\title{
Anti-mémoires. Noms, reflets et écritures
}

Countermemoires. Names, reflections and writings

\section{Filippo Fimiani}

\section{OpenEdition}

\section{Journals}

Édition électronique

URL : http://journals.openedition.org/imagesrevues/3466

DOI : 10.4000/imagesrevues.3466

ISSN : 1778-3801

\section{Éditeur :}

Centre d'Histoire et Théorie des Arts, Groupe d'Anthropologie Historique de l'Occident Médiéval, Laboratoire d'Anthropologie Sociale, UMR 8210 Anthropologie et Histoire des Mondes Antiques

\section{Référence électronique}

Filippo Fimiani, «Anti-mémoires. Noms, reflets et écritures », Images Re-vues [En ligne], Hors-série 5 | 2016, mis en ligne le 01 janvier 2014, consulté le 30 janvier 2021. URL : http://

journals.openedition.org/imagesrevues/3466; DOI : https://doi.org/10.4000/imagesrevues.3466

Ce document a été généré automatiquement le 30 janvier 2021.

Images Re-vues est mise à disposition selon les termes de la Licence Creative Commons Attribution Pas d'Utilisation Commerciale 4.0 International. 


\section{Anti-mémoires. Noms, reflets et écritures ${ }^{1}$}

Countermemoires. Names, reflections and writings

Filippo Fimiani

Les miroirs feraient bien de réfléchir un peu plus avant de renvoyer les images.

Cocteau, Essai de critique indirecte, 1932

Reflection is for mirrors. Can't you just hand me a

report card?

Sherlock à Watson, Elementary, Season 1, Episode

12,2013

\section{Faire écran}


1 Les liens ordinaires et singuliers, les partages surprenants et indécidables entre des formes de vie historiques, des textes et des images sont, en même temps, sensibles et idéologiques, phénoménologiques et herméneutiques, esthétiques et rhétoriques. Ils sont tels justement puisqu'ils sont affectés par la répétition, c'est-à-dire par plusieurs figures, économies et politiques du temps,

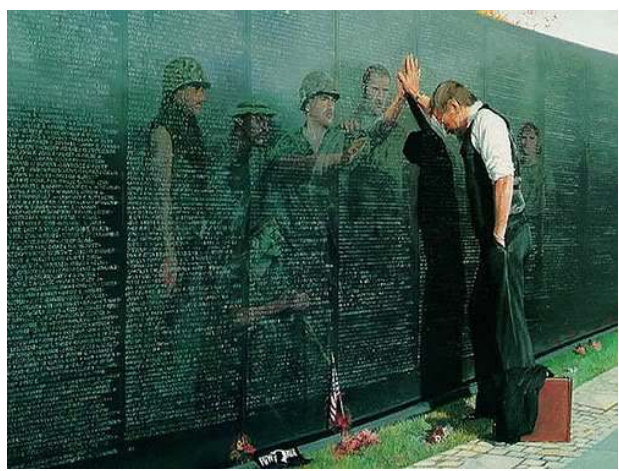
individuel et collectif, local, national ou globalisé.

2 Dispositifs médiaux et mnésiques par excellence, les monuments incarnent et supportent en pleine figure ces affections temporelles. Personnels ou communs, secrets ou publics, encryptés ou élevés, ils ont en fait une double destination. D’une part, les monuments véhiculent l'automatisme symbolique du temps officiel et partagé de l'histoire collective, qu'il soit discursive ou iconique et iconographique; de l'autre, ils sont intermédiaires $\mathrm{du}$ hasard du temps psychique singulier et de l'expérience perceptuelle réelle de chacun. Les répétitions codifiées des rituels identitaires communautaires sont en effet structurellement médiées et médiatisées aussi par les retours d'un réel, refoulé ou intégré dans le présent, par des revenants qui, arrivant après-coup, font régresser et antidatent, ou anticipent et projettent au-delà, les contextes d'usage de l'histoire collective.

3 La citation par laquelle je vais donc introduire et aborder la question d'une politique et peut-être d'une apocalypse en suspens, d'une crise en réserve de la mémoire et de ses média, de ses parerga spectraux et fantasmés -, je l'extrais d'un article ${ }^{2}$ consacré justement à un monument public : le Vietnam Veterans Memorial de Washington. Pour l'auteur de l'article, paru pendant l'été du 1985 dans "The Nation ", le philosophe Arthur Coleman Danto, l'œuvre d'art public de Maya Lin est sans doute non-figurative, mais profondément représentationnelle - étant donné, affirme-t-il, la «nature textuelle de l'art mémorial (de tout art, s'il est vraiment tel)». Une phrase à la page suivante relance et résume ainsi l'argumentation : "tout ce qui concerne le Mémorial, fait partie d'un texte ».

4 Avant d'expliquer le lexique à la fois apparemment friendly et pourtant quelque peu obscur de Danto - toute sa théorie de l'Aboutness y est condensée -, juste quelques mots ${ }^{3}$ sur le VVM. (Fig.1) 
Figure1

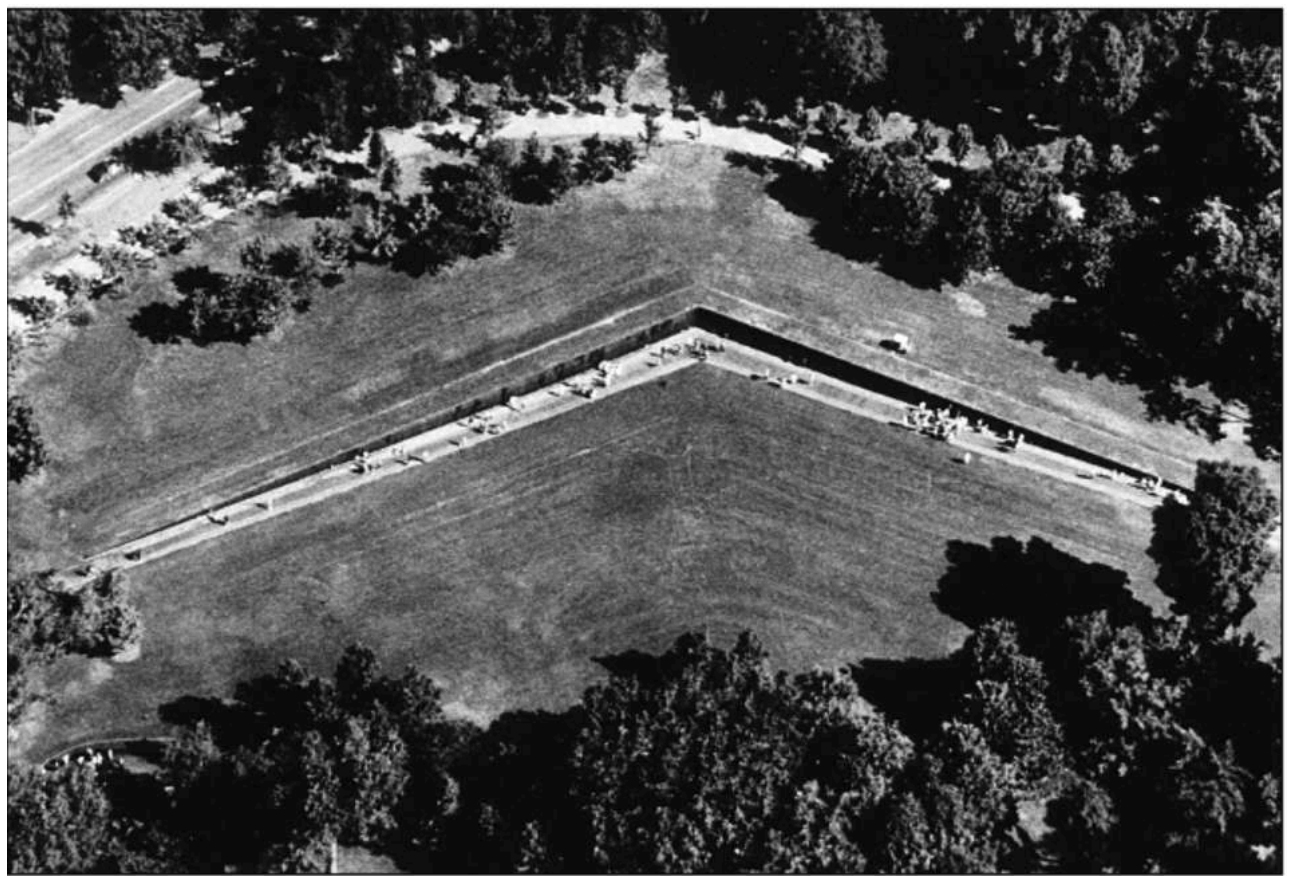

Maya Lin. Vietnam Veterans Memorial, 1982, Washington.

5 Le Mémorial fût inauguré officiellement le jour des Vétérans, le 13 novembre 1982, et il fut immédiatement entraîné dans une foule de polémiques violemment idéologiques, suite au prix du projet, choisi parmi quelques milliers de concurrents, et signé par une architecte alors encore inconnue. Certes, les formes du VVM sont très minimales, très peu monumentales, sinon anti-monumentales : deux murs de granite noire polie, qui coupent la terre et ouvrent comme un fossé, verticalement tranchant en profondeur le sol. (Fig.2) 


\section{Figure 2}

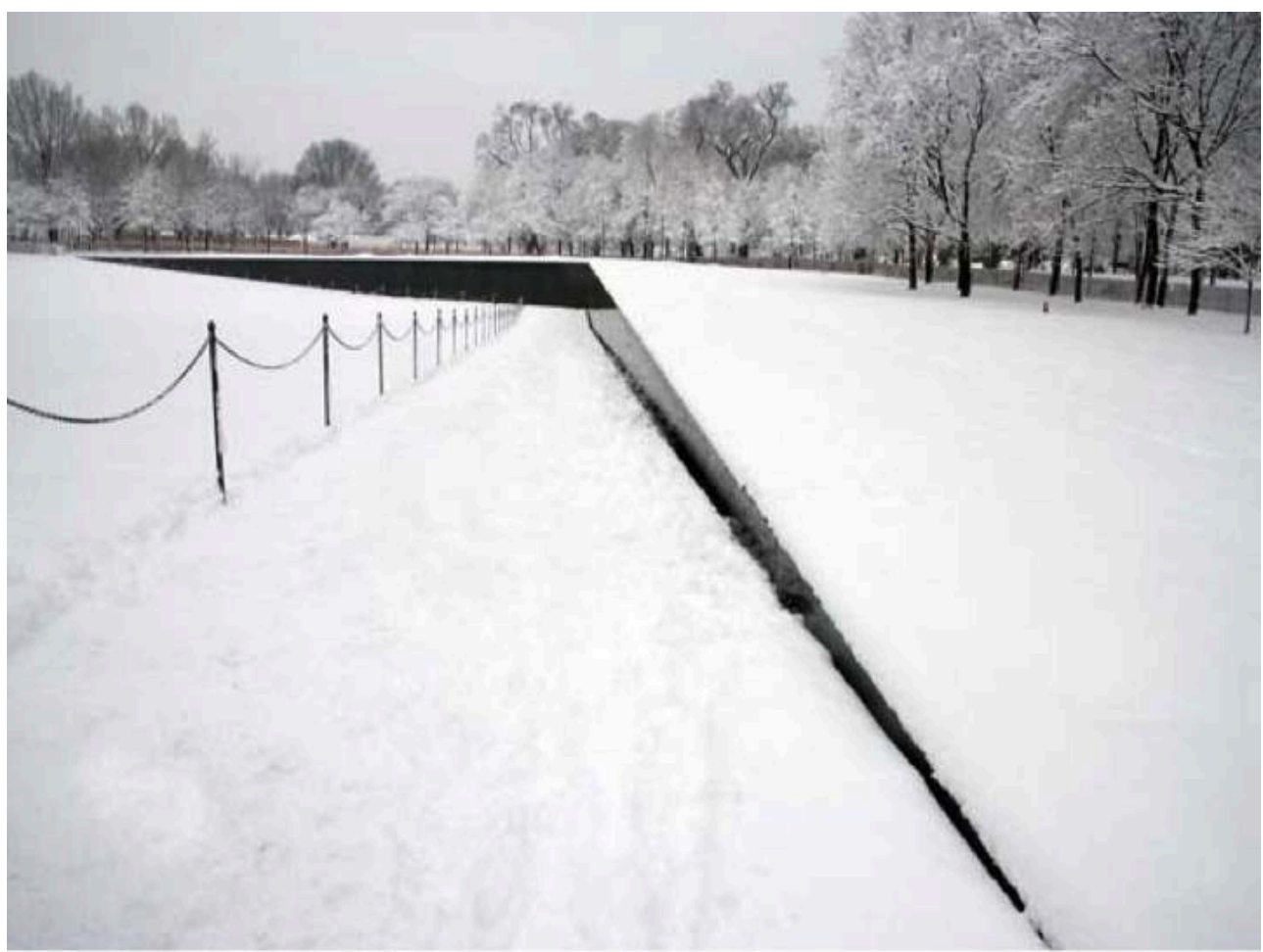

Maya Lin. Vietnam Veterans Memorial, 1982, Washington

Ce triangle isocèle - trois mètres au point le plus haut, vingt centimètres au plus bas - , on pourrait le décrire comme semblable à un boomerang, à une chauve-souris, ou à un monstre venu de Mars. (Fig.3) 
Figure 3

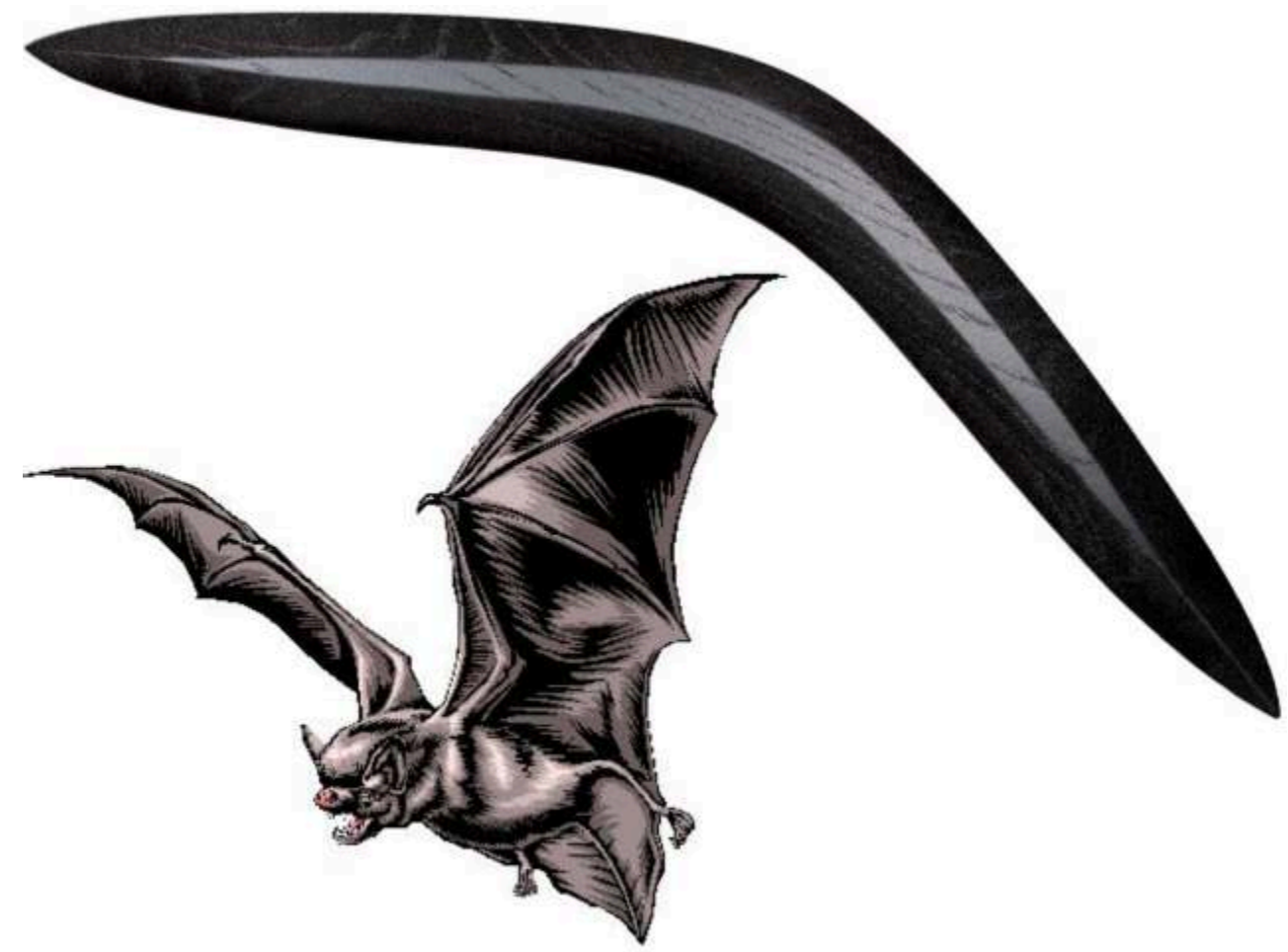

Boomerang, chauve-souris.

7 La «surface plate, pure» du Mur, selon les mots de Maya Lin en personne - assez proches de la Flatness et de la Purity du Modernisme et du Minimalisme, même si l'architecte a souvent affirmé avec détermination que son œuvre n'est pas intransitive et autoréférentielle, mais qu'elle se réfère à ce sujet singulier qui est la guerre du Vietnam avec ses morts nord-américains -, a provoqué toute une rêverie par ses aspects et ses formes. A la fois artistique et populaire, pour ainsi dire, un démon ou un fantôme analogique s'élance du Mur, va et vient entre les objets du loisir sportif, entre les bestiaires du gothique et de la science-fiction, entre les formules architecturales de l'art public confondues avec le vocabulaire formel des grands immeubles de n'importe quelle périphérie urbaine nord-américaine. Bien que constitué d'une simple surface lisse marquée par 58000 noms, sans aucune indication de rôle et rangés suivant un simple ordre chronologique, le Mur a convoqué un imaginaire visuel assez flottant et superficiel. Il mêle pop et culture de masse, qui va de Bram Stoker ou Frank Miller (qui au début des années quatre-vingt donne une nouvelle vie à Batman, le héros de Bob Kane et Bill Fringer) (Fig.4), à Richard Serra (Fig.5) sur lequel je m'attarderai, au monolithe de Stanley Kubrick (Fig.6) et aux Planks de John McCracken (Fig.7). 
Figure 4

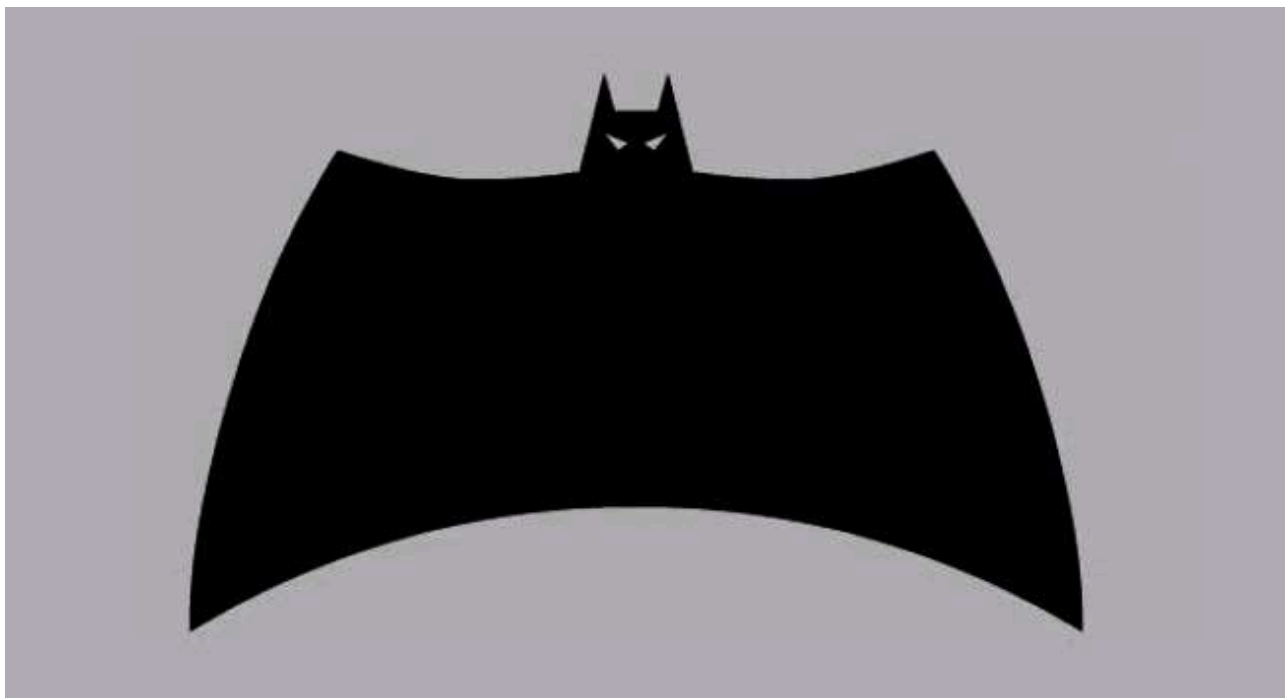

Logo de Batman, Batman and the outsiders, D.C. Comics, Aout 1983, écrit par Mike W. Barr, illustré par Jim Aparo.

\section{Figure 5}

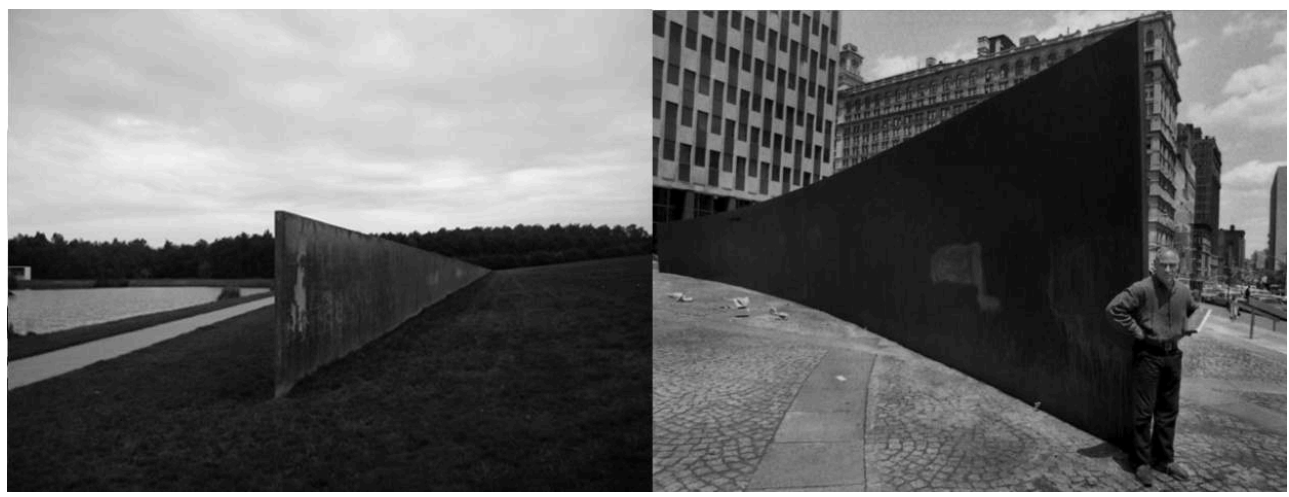

Richard Serra, Sealevel, 1989-1996, De Wetering, Pays-Bas ;Tilted Arc, 1981-1989, Federal Plaza, New York.

\section{Figure 6}

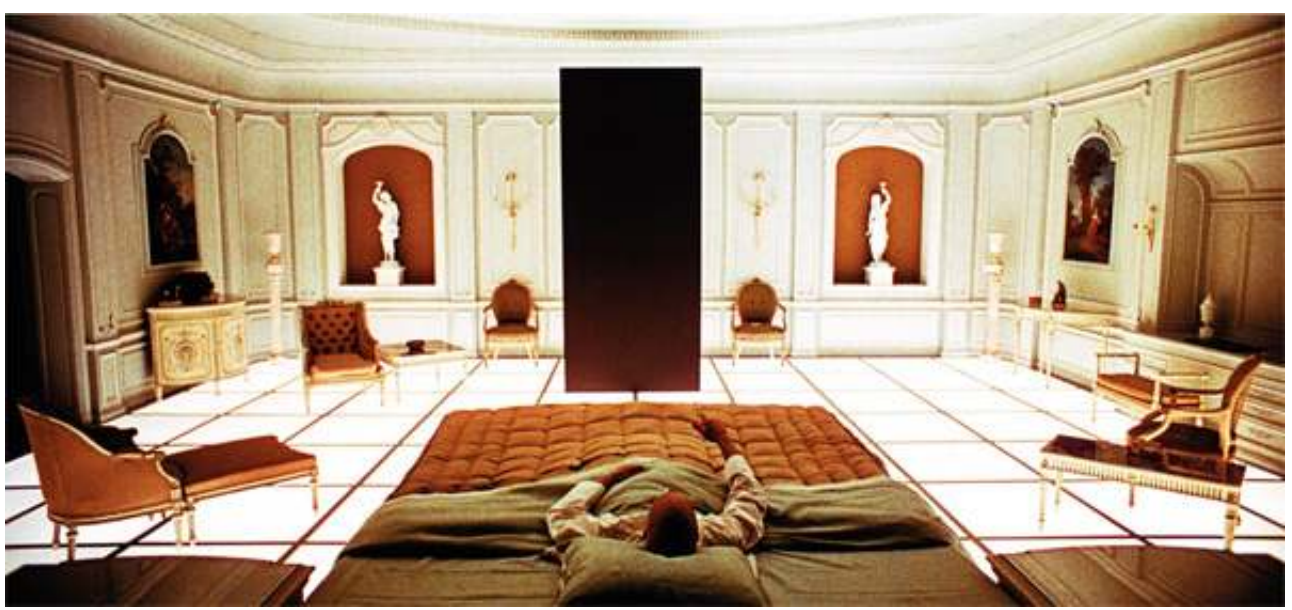

Stanley Kubric, Space Odissey, 1968. 
Figure 7a

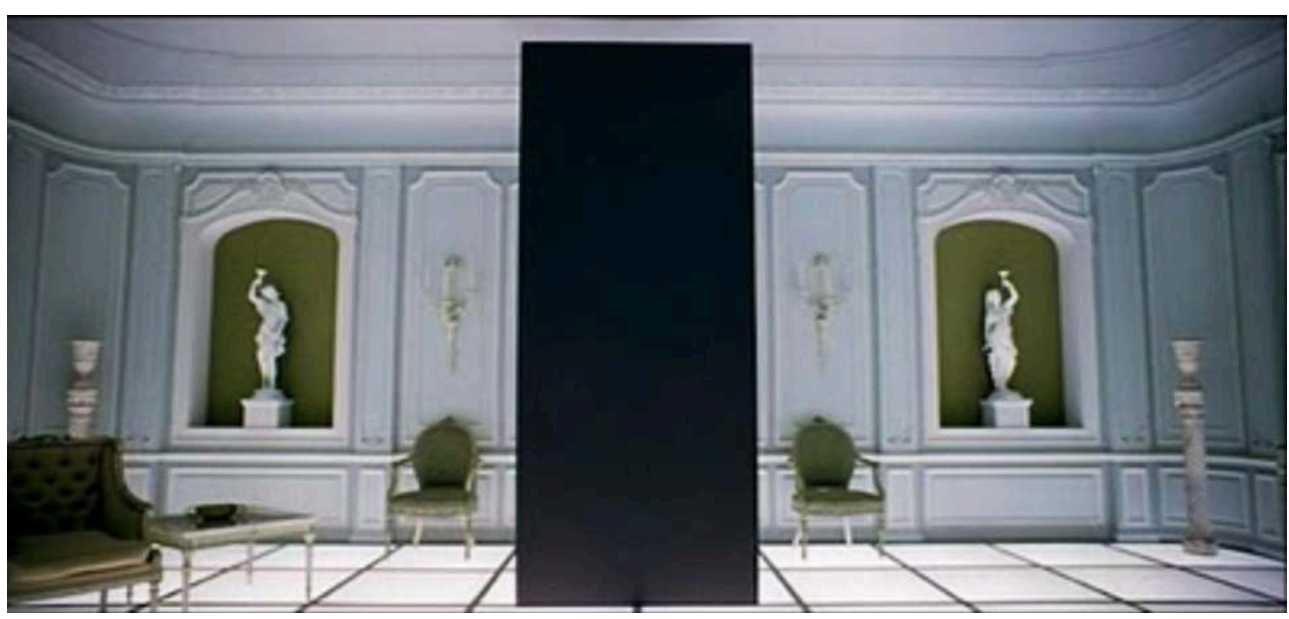

John McCracken, Black Plank, 1967.

\section{Figure $7 \mathrm{~b}$}

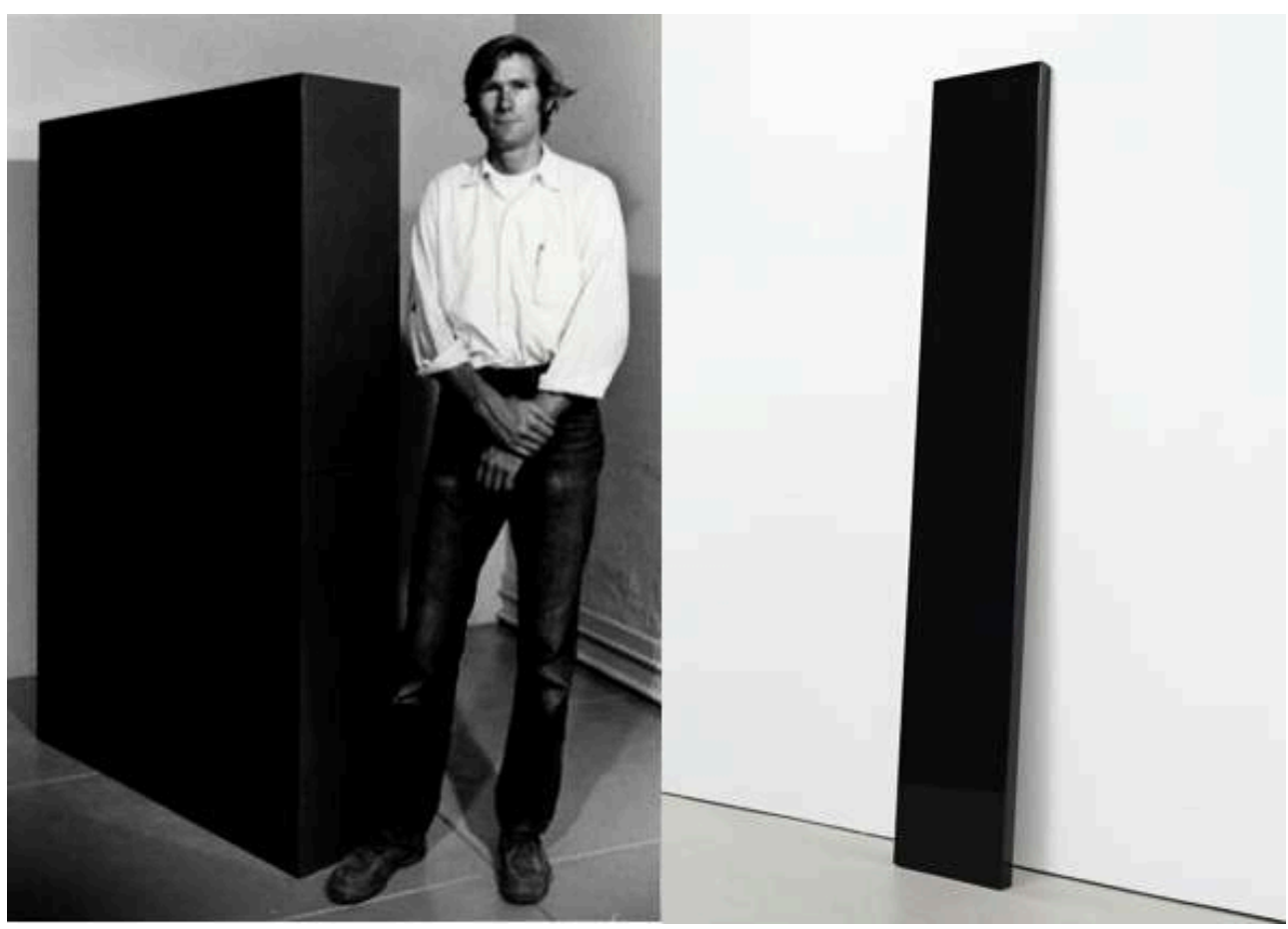

John McCracken, Black Plank, 1967, et photo de Frank J. Thomas de l'artiste dans son atelier à Costa Mesa, California, vers le 1966.

Certes, on pourrait comprendre que l'absence de repères iconographiques monumentales classiques et la pléthore de suggestions formelles assez superficielles repérables dans la culture visuelle de masse, puissent troubler et déranger la reconnaissance aspectuelle minimale par les visiteurs du Mémorial, qui ne sont pas nécessairement connaisseurs des affaires du monde de l'art et, accablés et émus, ont des attentes et des attitudes radicalement différentes, sans doute plus émotionnelles et irréfléchies qu'intellectuelles et savantes-ou cool. Face à un artefact destiné à perpétuer la mémoire nationale, et engagé dans une pratique collective du deuil, le 
public des visiteurs n'est, au moins en principe, composé que des gens ordinaires, fort attristés et dépourvus, ou détachés de toute compétence ou posture esthétique spécifique. Selon les adversaires de Maya Lin, c'est précisément cela un point critique assez important : même si partielle et temporaire, cette condition de non-familiarité et de non-reconnaissance face aux formes quasiment abstraites et non-monumentales du VVM troublerait les attentes perceptuelles et pragmatiques habituelles et historicisées et, finalement, introduirait un sentiment de déplaisir moral et un désaccord identitaire. Selon cette perspective, le Mur aurait fait écran aux visiteurs, il aurait interdit l'accès à sa fonction et signification culturelle, sociale et politique.

9 Pour combler et dépasser la pauvreté extrême du lexique formel et iconographique du Mur, le parti réactionnaire et conservateur a ainsi obtenu, malgré les protestations personnelles de Maya Lin, d'intégrer d'autres éléments fort réalistes et mimétiques. On a donc implanté deux groupes plastiques en face du VVM, tous les deux en bronze et de dimensions naturelles: (Fig.8) le premier, réalisé en 1985 par Frederick Hart, Three Fighting Men ; (Fig.9) le deuxième, posé en 1993, le Vietnam Women's Memorial, fait par Glenna Goodacre.

Figure 8

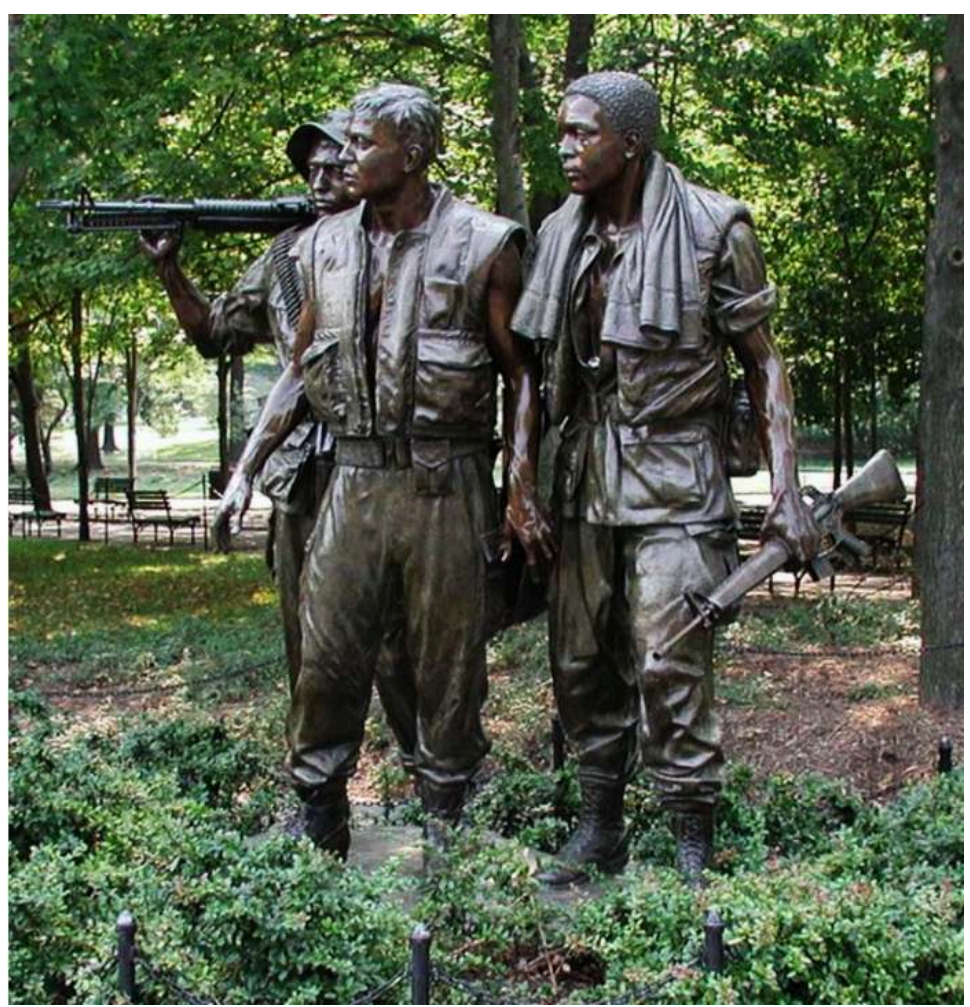

Frederick Hart, Three Fighting Men, 1985, Washington. 
Figure 9

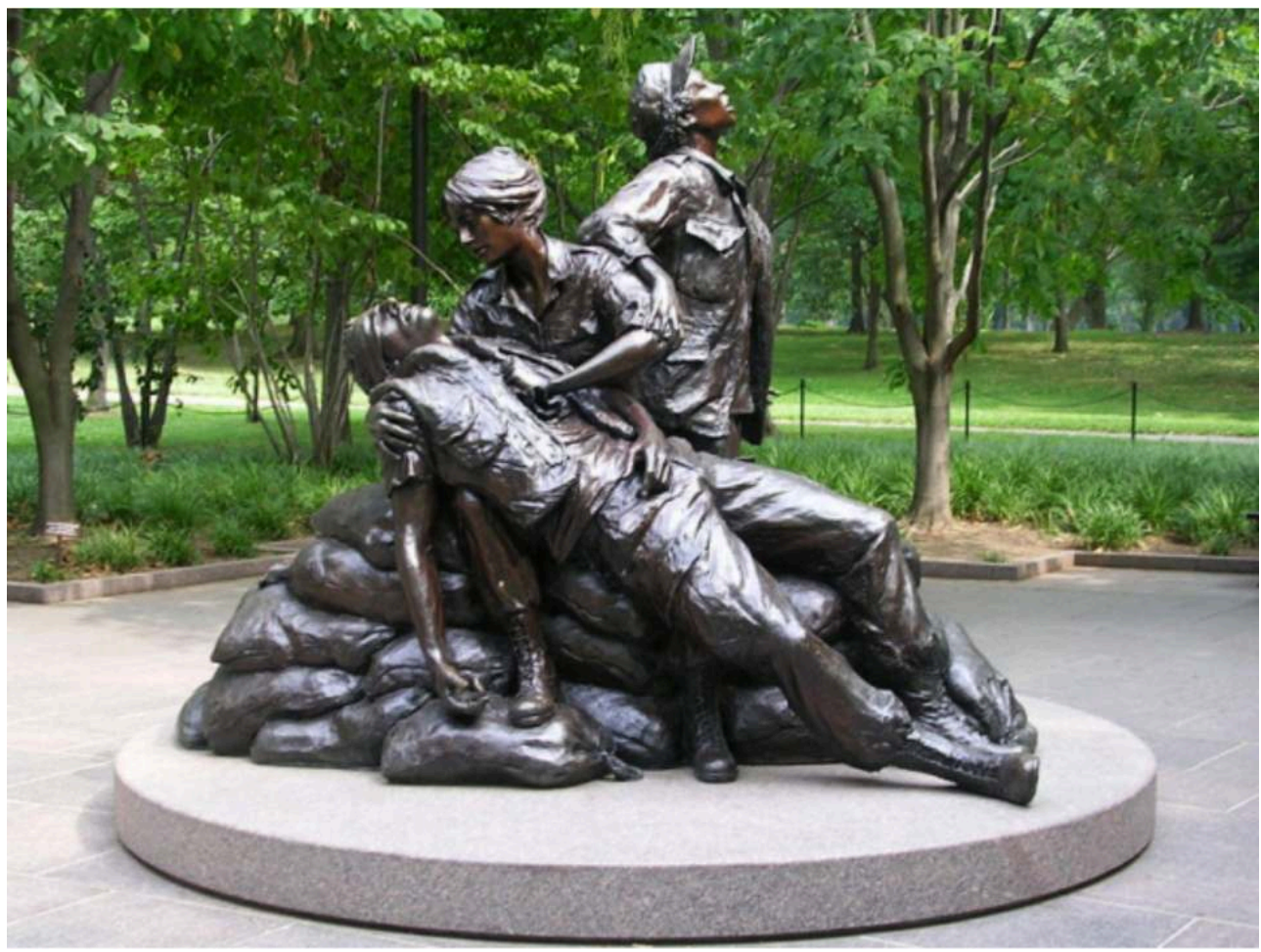

Glenna Goodacre, Vietnam Women's Memorial, 1993, Washington.

Or, les argumentations contre le Mémorial ont manqué l'articulation réelle et vivante entre la matérialité perceptible de l'objet, le cadre environnemental, le contexte d'usage et l'horizon d'attente des visiteurs, avec leurs habitudes perceptuelles et leurs préconcepts sur l'art public contemporain, leurs prédispositions émotives et pragmatiques. Le préjugé négatif sur le succès du Mur, concentré sur son étrangeté esthétique par rapport au canon monumental et fixé sur une réception standard, plate et entièrement focalisée sur les propriétés esthétiques, a raté la possibilité d'une nouvelle configuration véritablement historique, à la fois versatile et stable, entre l'habitus et l'aisthésis, entre le social et l'esthétique. Et c'est justement cette articulation active dans le cadre d'un espace public, que la communauté éthique des visiteurs, en pèlerinage des tous les coins des Etats-Unis, a réalisé et ne cesse de perpétuer encore aujourd'hui. En 2007, le Mémorial est dixième dans la List of America's Favorite Architecture rédigée pour le cent cinquantenaire de l'American Institute of Architects. Les détracteurs du Mur ont en somme sous-estimé qu'une forme monumentale si peu traditionnelle et héroïque pouvait incorporer, en tant que médium matériel extériorisé, une mémoire collective vivante et donner suite à un accord esthétique fortement identitaire et positif et à une beauté éthique admise et adoptée par le sens commun. L'extraordinaire popularité du Mémorial est par ailleurs citée par Danto parmi les preuves concrètes et empiriques de son hypothèse philosophique sur l'art public. L'œuvre anti-monumentale de Washington exemplifie ce qu'il appelle, à la suite de la pulchritudo adherens de Kant et, malgré l'apparent contraste de dictionnaire, d'accord avec la positivité factuelle et historique d'Hegel, la « beauté interne » de l'art. 


\section{Incorporations, modes d'emploi}

11 Qu'est-ce que internal Beauty? Et comment, selon l'énoncé de Danto, fait-elle partie du texte et donc du lisible, plutôt que du visible?

12 Dans son livre majeur, La transfiguration du banal, le philosophe américain propose une esthétique de la signification au détriment d'une esthétique de l'aspectualité : c'est la transfiguration sémantique et non pas l'appréciation perceptuelle, agréable ou pas, qui joue un rôle central dans la définition, l'interprétation et la compréhension d'une œuvre d'art. Selon ce parti pris du textuel, les propriétés artistiques sont à part entière sémantiques et, comme telles, incorporées dans l'œuvre - plus précisément, elles sont embodied Meanings. Dans L'Abus de la Beauté, livre sorti en 2003 après un cycle de conférences, et qui approfondit justement la question de la beauté - l'un des thèmes marginalisés dans les travaux des années précédents, devenu par la suite de plus en plus urgent, jusqu'à What Art $i s^{4}$, sorti juste avant sa mort-, Danto convoque précisément le Mémorial.

Quelle que soit l'explication de la beauté ressentie du Mur, écrit-il's, elle ne se comprend que par référence à la «pensée ». Elle fait partie de la signification de l'œuvre. [...] Dans le Mur, la pensée appartient à l'œuvre et explique la beauté. Dans la beauté naturelle, la beauté est externe à la pensée; en art, elle est interne à l'œuvre.

13 Cette définition de la beauté interne mobilise plusieurs sous-textes, notamment philosophiques. En fait, Danto fabrique cette définition du beau à partir de Kant et contre lui, plus précisément contre le pseudo-kantisme de Clement Greenberg ${ }^{6}$. Est visée son idée d'une peinture finalement autoréflexive et autoréférentielle, qui ne signifie strictement rien et qui, plate, plaît pourtant, comme la pulchritudo vaga des arabesques naturels et artistiques chez Kant, qui active un plaisir de la réflexion tout en étant sans concepts déterminés, selon les analyses célèbres des paragraphes 14 et 16 de l'Analytique du beau de la Critique de la faculté de juger. Par ailleurs, Danto avait déjà mis au point une différence de taille entre l'art et l'art public, notamment à partir de la question très délicate du rapport entre la beauté et la signification, ainsi qu'entre les formes et les usages. Une fois de plus, me semble-t-il, il s'agit d'une suite à l'identification kantienne de la «chose principale» de l'architecture, notamment monumentale et publique, avec l'usage. Dans deux articles pour « The Nation », publiés le 22 juin 1985 et le 19 avril $1986^{7}$, et sans que ce soit un hasard, il avait choisi une fois de plus le Mur de Maya Lin comme l'exemple le plus éloquent, voire comme véritable paradigme et modèle.

Or, tout modèle a un contre-exemple, voire un principe de falsifiabilité euristique : pour le VVM, (Fig.10) c'est Tilted arc de Richard Serra. 
Figure 10

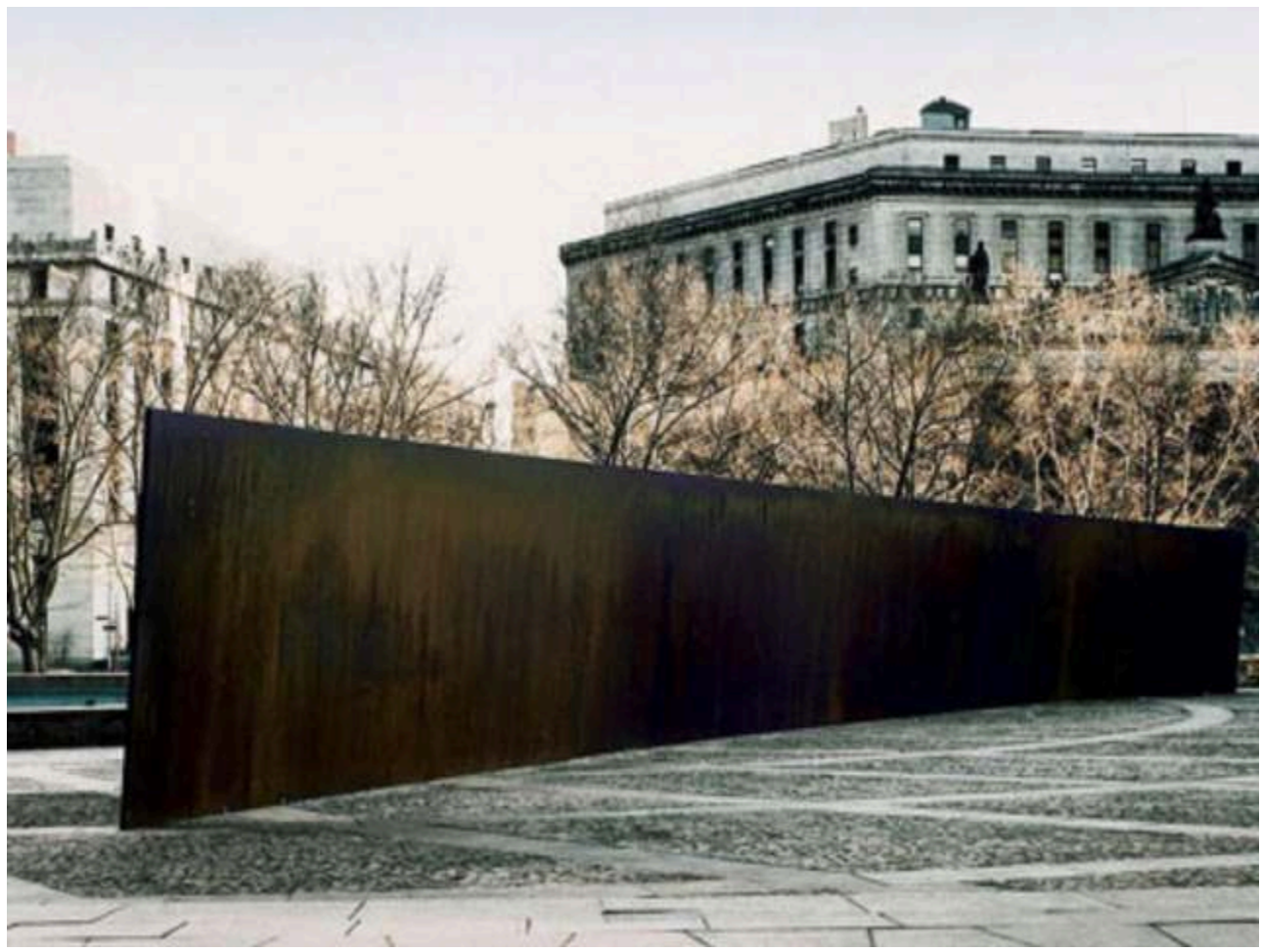

Richard Serra, Tilted arc, 1981, Federal Plaza, New York.

Le philosophe newyorkais s'était en effet engagé dans une campagne de protestation assez virulente, qui avait mené en 1989, après une bataille juridique très âpre, à l'enlèvement et à la destruction de l'œuvre disputée du plasticien californien. La sculpture de Serra, conçue comme site-specific, trois mètres de hauteur, trente-six de longueur, placée au cœur de Federal Plaza à New York, était en acier Corten non traité, rapidement couvert de rouille et taché de graffiti et empreintes, comme d'autres sculptures, telle Clara-Clara aux Tuileries (Fig.11) - pourtant déplacée ailleurs sans problèmes. Inversement à la surface inaltérable, polie et réfléchissante en marbre noir du VVM, le mur de Serra, bien que permanent et de grandes dimensions, était vite altéré et presque devenu délabré. 
Figure 11

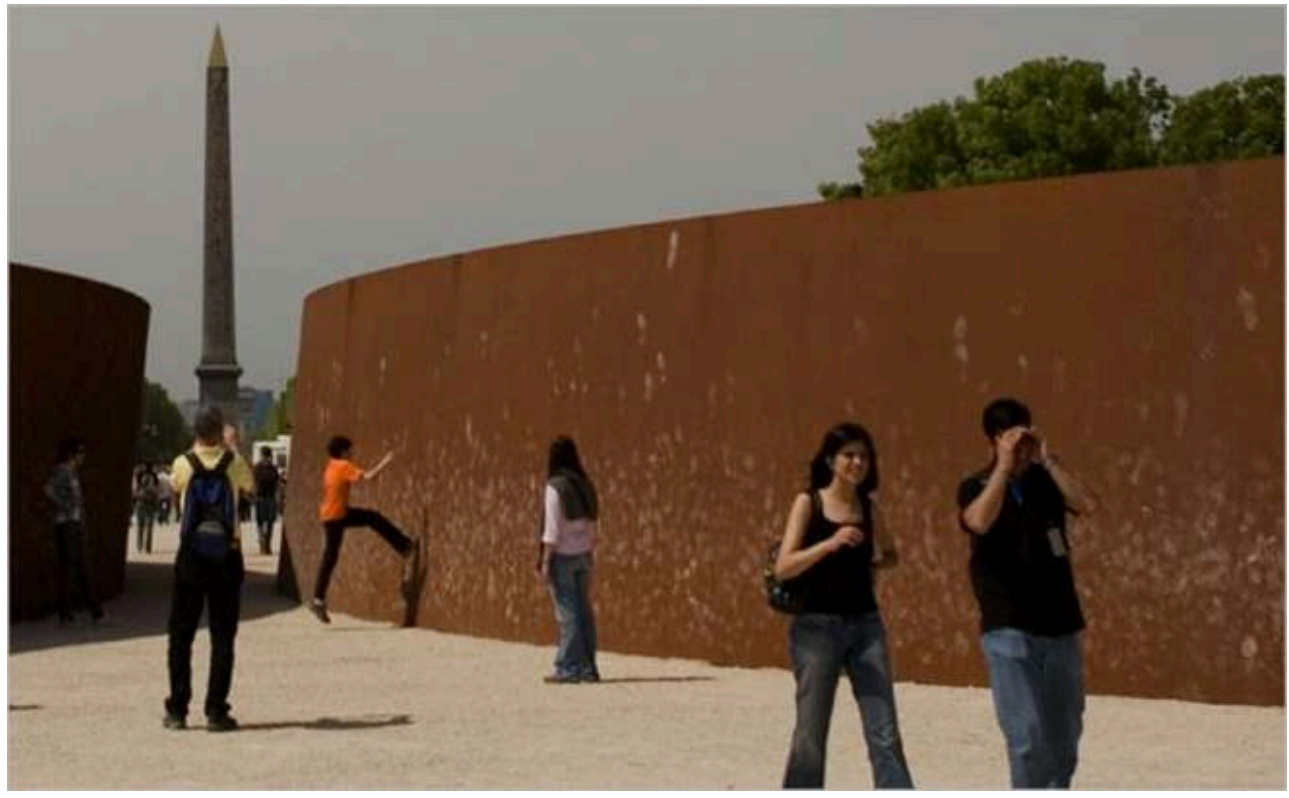

Richard Serra, Clara-Clara, 2008, Tuileries, Paris.

En d'autres termes, il n'était pas perçu et décrit comme un monument, dont il n'avait finalement pas acquis le statut ontologique et l'étiquette définitionnelle ${ }^{8}$.

17 Selon Danto, on aurait pu sans aucun doute apprécier Tilted arc en tant qu'œuvre d'art. Et cela pour diverses et bonnes raisons. Pour ses qualités matérielles et formelles visibles; pour ses qualités métaphoriques ou symboliques interprétables; (Fig.12) pour les qualités typologiques de son dessin, bien lisibles, en planimétrie ou photographie prise d'en haut, par rapport à l'échelle urbanistique. 
Figure 12

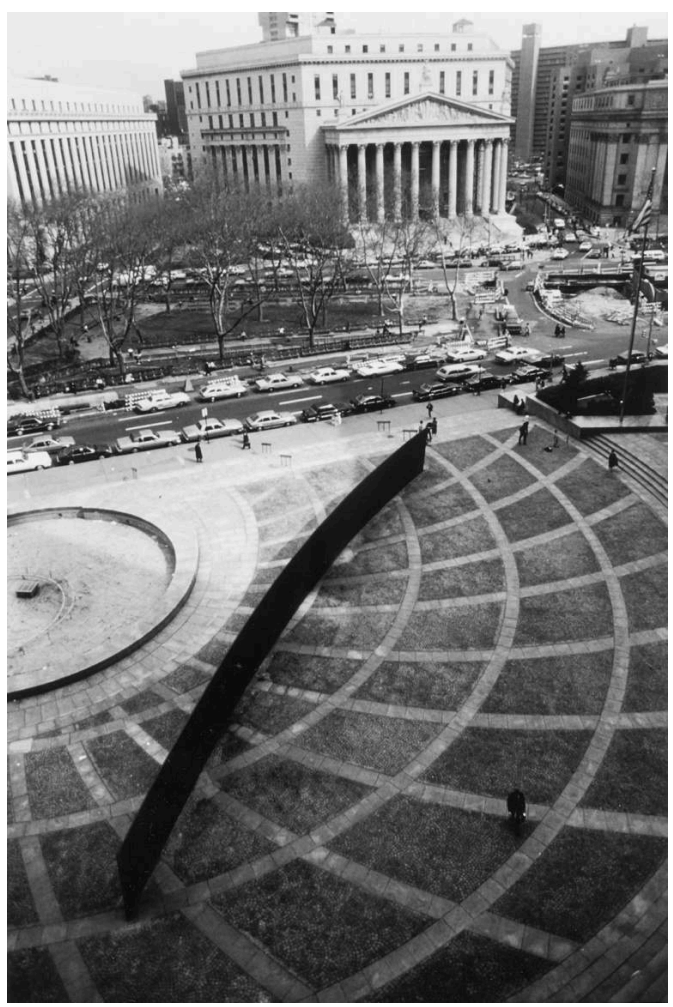

Richard Serra, Tilted arc, 1981, Federal Plaza, New York.

18 Autrement dit, on aurait pu jouir perceptiblement et intellectuellement soit de la présence réelle de l'objet avec ses propriétés esthétiques physiques; soit de sa capacité à donner une "nouvelle identité plastique " à l'espace et au lieu, à modifier les parcours routiniers des passants pris par le train-train de la vie de tous les jours ; soit encore de sa fonction morphologique, en tant qu'il est une esquisse préparatoire ou un dessin remémorant, voire un "écho" graphique comme dit Yves-Alain Bois, d'un temps révolu devenu vestige ineffaçable, (Fig.13) une cicatrice sur le tissu urbain survenue suite à un acte de violence et de destruction. 
Figure 13a

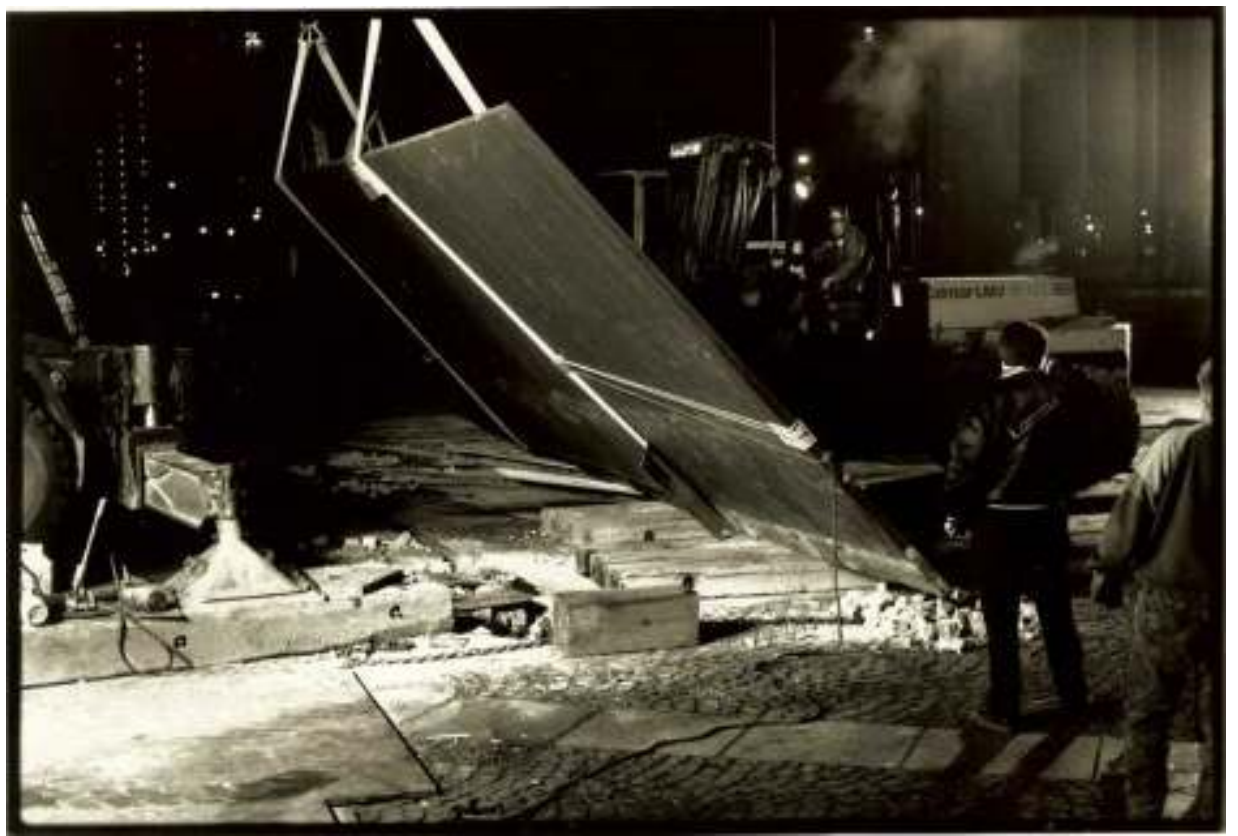

Richard Serra, Tilted arc, 1981, Federal Plaza, New York.

\section{Figure 13b}

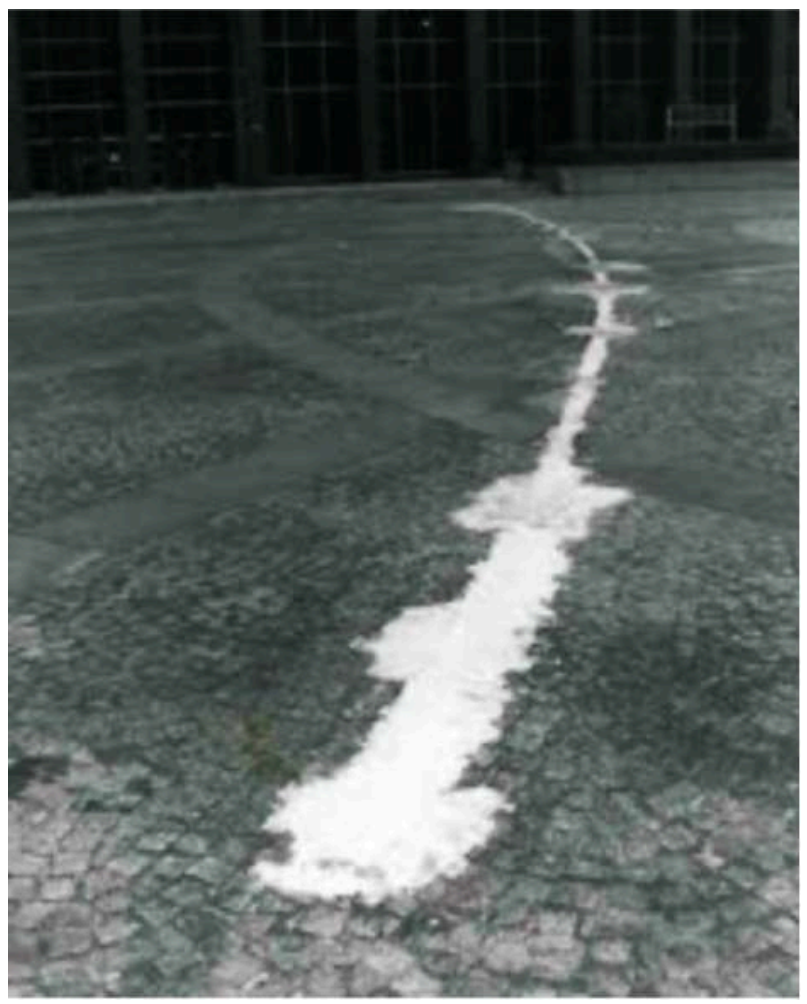

Richard Serra, Tilted arc removed, 1989, Federal Plaza, New York.

19 Et pourtant, d'après Danto, la réussite de Tilted arc en tant qu'œuvre d'art n'a rien à voir avec son échec en tant qu'œuvre d'art public. Comme telle, elle aurait dû se rapporter aux pratiques quotidiennes des habitants et des usagers du lieu. En tant qu'œuvre d'art 
public, elle aurait dû correspondre à l'éthos, c'est-à-dire aux habitudes et aux manières quasiment automatiques du monde de la vie ordinaire, et non pas s'inscrire dans le logos, c'est-à-dire dans les discours spécifiques et les interprétations spécialisées du monde de l'art. La bonne compréhension de l'œuvre englobe donc soit la pure visibilité du seul aspect, soit la lisibilité restreinte du seul texte: elle concerne finalement la place de l'œuvre dans une forme de vie historique et routinière et les relations qu'elle peut activer dans un contexte humain donné.

La nature textuelle du Mémorial n'est alors pas saisissable par un acte de lecture comme celui du déchiffrement, par exemple, de la forme symbolique codifiée qu'est son croquis ou son projet de réalisation, ou son image photographique d'en haut. Danto n'est pas Goodman, et son textualisme est plus atténué, il est tempéré par un pragmatisme modérément historicisé, voire par une herméneutique assez traditionnelle, peut-être inavouée. A ma connaissance, il ne nomme jamais Hans-Georg Gadamer, mais il partage avec lui un héritage assez fidèle à Hegel. Au fond, Danto se limite à appliquer au Mémorial et à l'art public les consignes méthodologiques affirmées déjà à propos de la métaphore visuelle et textuelle : la critique ne peut que décrire le fonctionnement des métaphores internes à l'œuvre, y compris, dans le cas du VVM ou de Tiltled arc, leurs rapports avec d'autres éléments ou formes signifiantes dans l'espace physique réel. Certes, cette analyse ne peut jamais se substituer à l'œuvre comme telle ni avoir sa force. Contre une lecture déflationniste ou réductionniste, et contre toute simplification des métaphores langagières et visuelles, la critique doit donc consigner au destinataire les informations nécessaires pour pouvoir répondre comme il faut à la puissance sémantique de l'œuvre.

21 Si l'on reconnaît une position assez fidèle à l'esprit humaniste de l'iconologie classique et à sa philosophie de l'histoire téléologique, il est capital de bien comprendre le rôle exemplaire du Mémorial. Celui-ci ne tient pas ici la place du chef-d'œuvre dans un sens traditionnel, mais il exemplifie le fait que la force du beau, la vis de la beauté interne à l'œuvre, et de l'art public, est ressentie et pour cela elle est comprise. Pas très loin du Feeling for the Rules indiqué par Wittgenstein ${ }^{9}$, comme condition préliminaire à la production et surtout à la bonne réception de l'œuvre d'art et à sa signification sociale et culturelle, et carrément différencié du bare Feeling, du ressentir superficiel et éphémère, ce Feeling est donc lui-même profond et durable. Ce sentiment ordinaire est l'expression sensible, psychique et corporelle, de la sédimentation historique du sens, du Meaning incarné dans l'œuvre et partagé par une communauté donnée à travers les émotions et les sentiments les plus enracinés et identitaires. Comme Danto écrit dans The Transfiguration of the Commonplace, " il y a des choses que nous savons devoir ressentir [to feel] dans des conditions répondant à une certaine caractérisation [...] dans des conditions que tous les membres de notre communauté comprendront ${ }^{10}$. Autrement dit, suite à la réflexion d'Aristote sur les syllogismes rhétoriques, dits abrégés ou défectueux, et peut-être pas si loin de la philosophie du langage ordinaire de Wittgenstein, selon Danto il y a des précompréhensions tacites qui règlent l'ensemble des formes de la vie ordinaire, y compris les relations esthétiques aux œuvres d'art en général et les expériences identitaires collectives activées par les œuvres d'art public, notamment par les monuments.

Fidèle à ce principe d'une rhétorique élargie, l'article de Danto du 1985 s'ouvre par une sorte de typologie élémentaire de l'architecture monumentale publique ${ }^{11}$ : 
Nous érigeons les Monuments pour nous ressouvenir toujours, et nous construisons les Mémoriaux pour n'oublier jamais. [...] Les Monuments commémorent le mémorable et incorporent les mythes des origines. Les Mémoriaux ritualisent la remémoration et marquent la réalité des morts. [...] Très peu de nations érigent des Monuments pour leurs défaites, mais beaucoup dressent des Mémoriaux pour les vaincus décédés. Les Monuments font des héros et des triomphes, des victoires et des conquêtes des êtres perpétuellement présents et partie de la vie. Le Mémorial est une enceinte spéciale, exclue de la vie, une enclave ségrégée où nous honorons les morts. Avec les Monuments, nous honorons nous-mêmes.

Parmi les exemples cités, le Washington Monument et le Lincoln Memorial. Mais le paradigme d'un art public de la mémoire est justement le Vietnam Memorial, qui est anti-monumental sans être intransitif ni autoréférentiel. Le Mur, on l'a vu, n'est ni à lire d'une manière abstraite ou formelle, ni à expérimenter d'une manière sensorielle ou perceptuelle. La nature textuelle du «monument» public de Maya Lin n'est ni sémiotique ni somatique : elle est sémantique. Le Mémorial n'est pas, comme Tilted arc de Serra, site-specific et anti-environmental, et pour cela, anti-monumental, comme le suggère Dougles Crimp et comme le répète justement l'artiste californien ${ }^{12}$, puisqu'il suspend tout ancrage référentiel et toute croyance dans la notion, l'usage et l'histoire des monuments. Par conséquent, l'œuvre de Serra ne commémore rien, "not memorialize anything ». Au contraire, le Mur est ce que Platon appelait hypomnematon, c'est-à-dire un support mnésique matériel externe, un dispositif supplémentaire et plus durable de la mémoire individuelle interne, sensorielle et psychique. Comme tel, il fait partie de ce que Danto appelle, à la Hegel, un « paysage moral », c'est-à-dire un espace public d'une forme de vie entièrement historique où on partage et on transmet des valeurs identitaires. Déterminées par une politique et une idéologie de la mémoire, celles-ci sont, au moins en principe, tendanciellement universelles, ou universalisables. Si tout texte est imperceptible, comme disait Derrida, il est tout de même, dit Danto, intelligible.

\section{3. « Un texte mystérieux et non encore déchiffré »}

Danto affirme qu'une œuvre d'art, et plus encore une œuvre d'art public, est telle lorsqu'elle est le support ou le véhicule, pas nécessairement isolable et spécifique, d'une batterie de propriétés relationnelles bien plus que seulement perceptuelles. Ce sont des propriétés, ou plus précisément des significations, qu'il faut ressentir et interpréter correctement. Autrement dit, à l'aide d'une formule très lapidaire mais assez fertile pour réfléchir sur la nature et l'économie du dispositif médial : "L'art public est le public transfiguré : c'est nous, dans le médium de la transformation artistique $»^{13}$.

On pourrait rédiger une table des matières des conditions nécessaires à un tel fonctionnement d'une œuvre d'art : il faut que l'œuvre soit à propos de quelque chose, qu'elle signifie et concerne un sujet; il faut qu'elle soit une représentation-pas forcément figurative, visible ou tangible - de ce qu'on appelle faute de mieux un contenu; il faut qu'elle ait une histoire matérielle et immatérielle, intentionnelle et pragmatique avant et après sa production factuelle; il faut que cette histoire soit réalisée à un certain moment de l'historie d'une civilisation, voire de l'histoire du monde ou, dirais-je, de l'histoire globalisée, y compris de l'histoire de l'art ; il faut que l'œuvre appartienne à une tradition, pas seulement artistique, mais à une culture 
visuelle; il faut qu'elle ait des qualités stylistiques et expressives les plus efficaces et irremplaçables et, pour cela, capables d'activer les réactions, elles aussi les plus justes et adéquates, notamment les sentiments moraux, individuels et collectifs dans notre cas. Et ainsi de suite.(Fig.14)

Figure 14

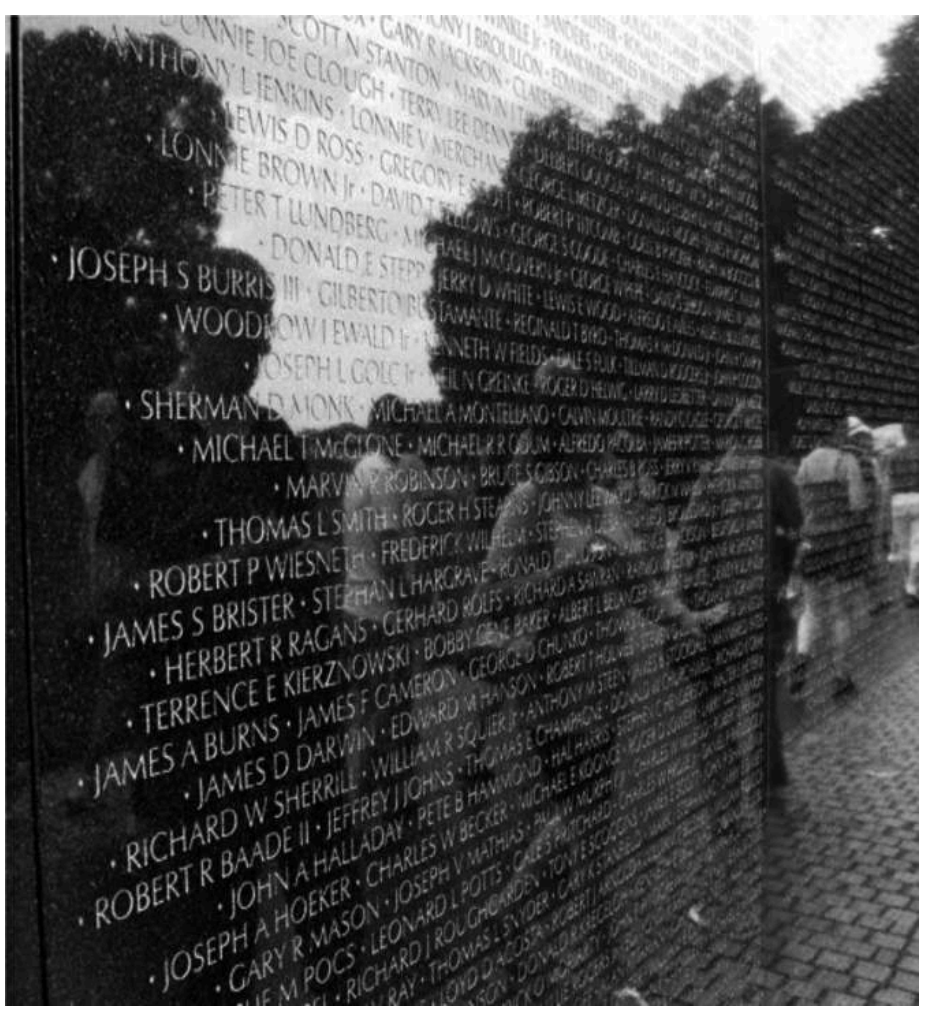

Maya Lin. Vietnam Veterans Memorial, 1982, Washington.

Suite à cette énumération assez incomplète de ces conditions, qu'on pourrait peut-être appeler transcendantaux esthétiques, relisons la première phrase consacrée au Mur dans The Abuse of the Beauty ${ }^{14}$ :

On ressent presque instantanément la beauté du Mémorial, puis elle est peut-être analysée et expliquée par référence à la manière dont les visiteurs (beaucoup d'entre eux viennent voir le nom de quelqu'un qu'ils connaissaient ou qu'ils aimaient et pour en faire un frottage et le remporter chez eux) voient leur reflet sur le même mur qui porte le nom des morts, comme s'il y avait une communauté des vivants et des morts, bien que la mort elle-même soit pour toujours.

Le sentiment esthétique, le Feeling du beau, n'est donc pas immédiat et irréfléchi. La beauté du Mémorial est "presque instantanément" ressentie, et ce petit et imperceptible délai n'est pas seulement phénoménologique. Il est déjà sémantique, c'est-à-dire qu'il ne concerne pas tellement l'hésitation visuelle, peu à peu ajustée et maîtrisée par le visiteur ému, voire bouleversé et confus, mais il exprime plutôt la temporisation culturelle qui structure les attitudes esthétiques et l'habitus éthique, pour ainsi dire la durée de la prise de corps des sentiments et des émotions face au beau moral. Ce négligeable retard sensible, pathémique, incarne l'ajournement cognitif nécessaire à une participation profonde à la forme de vie publique qui est le paysage moral, c'est-à-dire l'espace identitaire institué par le Mémorial. Ces manières de 
participation à l'espace public activées d'une façon exemplaire par l'œuvre de Maya Lin sont, selon Danto, depuis toujours historiques et sociales. C'est justement pour cela que la métaphore que toute œuvre d'art est, peut s'affaiblir ou devenir inaccessible, peut se détériorer ou dégénérer à cause des modifications des connaissances implicites et explicites nécessaires à la compréhension de sa signification. Elle peut aussi se détacher des règles de l'habitus auquel elle est destinée, s'émanciper des prémisses de son usage préconstitué, s'altérer et se libérer pour aller vers d'autres interprétations, pratiques ou expériences.

Certes, la beauté interne du Mémorial est, selon Danto, malgré tout liée à sa structure justificative rhétorique, à sa nature textuelle, qui est donc sans doute contextuelle et co-textuelle. Or, et c'est un point capital de ma lecture, cela signifie que la beauté du Mur de Maya Lin est toujours en réserve par rapport aux effets, aux sentiments, aux croyances et aux pratiques qu'elle pourrait produire. La beauté de l'œuvre d'art public est finalement en retrait face à son accomplissement et à son avenir. Sa nature, bien qu'historique et incorporée dans l'expérience vécue et ressentie du public, est aussi de l'ordre du possible et du potentiel, du devenir, pas seulement du prédéterminé, de l'accompli, de l'être. La nature non-naturelle, mais culturelle et sociale du texte, du contexte et du co-texte, qu'est le Mémorial, est donc relationnelle et virtuelle. Et cette nature s'actualise et se réalise par ce qui arrive et se produit: moins par les connaissances et les compétences données dans un jeu linguistique tout fait et enfermé sur lui-même, que par les pratiques ordinaires et les manières de vivre les perceptions, les émotions et les sentiments lorsqu'on visite le VVM et son lieu singulier.

Si tout ce à propos de quoi le Mémorial est, comme Danto déclare, « fait partie d'un texte ", ce texte, bien qu'il instaure une identité soigneusement, solennellement et souverainement repliée sur elle-même et sur sa tradition, est en même temps la manifestation d'une "nature » ou d'une "origine » opaque, peut-être enfouie, sans doute plus (ou pas encore) lisible comme telle. Avec Nietzsche et Foucault ${ }^{15}$, suivant l'usage allemand, je propose d'y voir une duplicité essentielle: l'« origine », ou, en allemand, l'Ursprung, du texte que toute œuvre d'art, notamment public et mémoriel, est en même temps Herkunft, c'est-à-dire « source » et " souche », et Entstehung, mot qui dénote au contraire l'« arrivée » et l'« apparition ». L'origine du texte du Vietnam Veterans Memorial, justement assumé par Danto en tant que type idéal et paradigme de l'art mémorial public et de tout art, est en fait double, voire plurielle. D'un côté, elle est "la souche et la provenance " inavouées et cachées de l'identité collective et nationale qui s'institue grâce au monument et s'incarne en lui, en y inscrivant son histoire canonisée comme ininterrompue et close, mais en réalité discontinue et marquée par des violences et blessures jamais apaisées. De l'autre côté, l'origine du texte du Mémorial s'expose comme "l'émergence, le point de surgissement [...] d'une apparition " singulière, comme "l'entrée en scène des forces » en lutte les unes contre les autres, comme un «théâtre sans lieu » où s'affrontent des différends qui n'appartiennent pas au même espace et au même temps. Une apparition d'une altérité radicale, donc: une réapparition du passé traumatique de la guerre et de la mort, qui résiste aux symbolisations et aux pratiques rituelles de la mémoire collective-et par l'intermédiaire du réseau numérique -, grâce auxquelles une communauté éthique s'institue au présent et se réalise comme identité en acte et sans failles.

Suite aux analyses lumineuses de Foucault que je viens d'évoquer, il me semble que, différemment de celui porté par Danto, un autre regard, en même temps 
phénoménologique et généalogique, doit s'attarder sur la surface réfléchissante du Mur. Selon cette autre manière de voir et de lire, cette surface plate et polie est une table de présentation traumatisante du passé - qui ne cesse pourtant de revenir - dans le présent du rituel collectif de deuil, et non pas un tableau commémorant d'(auto-)représentation identitaire, déjà préétablie et prédestinée. Dans cette exhibition spectrale, toute une contre-politique de la mémoire à l'état virtuel est donnée au visiteur ; c'est à lui d'en tirer parti et de la réaliser dans le domaine sensible commun, dans ce que Hannah Arendt appelle die Öffentlichkeit, l'espace public, qui est forcément esthétique.

\section{Un miroir noir}

31 Le texte qu'est le Mémorial est donc, et malgré lui-même, le "texte mystérieux et non encore déchiffré» évoqué par Nietzsche dans un fragment de La Volonté de Puissance ${ }^{16}$. Il est aussi, contre sa lisibilité idéologique manifeste, ce texte "dont le sens se révèle de plus en plus » aux spectateurs et aux « interprètes et [aux] augures pleins d'astuce » des choses nord-américaines et mondiales. Cette autre lisibilité qui apparaît avec une marge d'ambiguïté, de dissidence, de résistance, bref de liberté et d'indécidabilité, elle est épiphanique, à la fois encore à venir et rétrospective, prophétique et généalogique. La figuration momentanée qui clignote sur la surface miroitante du Mémorial elle est donc dialectique au sens de la « dialectique à l'arrêt » de Benjamin ${ }^{17}$ : en tant qu'image immobilisée et aléatoire, elle montre ici et maintenant une connaissabilité différente, elle manifeste l'instance même d'une lisibilité alternative, qui détourne et désarticule la nature textuelle du monument doublement prédéterminée par les précompréhensions et les pratiques de la communauté éthique donnée. On a vu que, dans ce cadre d'attentes accordées, à la fois rhétorique et historique, le Vietnam Veterans Memorial est à la fois un monumentum et un memento: il est un signe matériel qui doit monere et admonere, tantôt être un rappel magistral à la mémoire des victimes de la guerre, tantôt alerter sur le péril de la répétition toujours possible des horreurs et des défaites. Selon Danto, la beauté de la surface plate et brillante du Mur est fondée sur une structure syllogistique qui repose sur des prémisses éludées et pourtant ténues pour certaines, elle est une sorte d'évidence enthymématique sensible de cette double signification caractéristique de tout monument et mémorial public et privé. Ressentir la force de la remémoration et de la prévention incarnées dans le Mémorial dépasse tantôt une simple expérience esthétique, tantôt une commotion privée et du pathos ineffable et individuel. Pour le philosophe newyorkais, c'est la coappartenance à une forme de vie historique donnée et à son horizon non thématisé de croyances et de procédures, d'habitudes et pratiques y compris émotives, qui est la condition véritablement nécessaire pour une expérience esthétique sociale moralement et politiquement correcte.

Or, cette coappartenance est à la fois tendanciellement universelle et inéluctablement historique, je l'ai déjà dit et là je serais tenté de dire qu'elle est symptomatiquement nord-américaine et pop. Au nom de son pragmatisme historiciste, Danto rappelle quelques précédents architecturaux illustres, voire des icônes hyper médiatisées de la culture occidentale, (Fig.15) tel le Monument funèbre à Marie Christine d'Autriche (1798-1805) de Canova, ou Saint-Pierre. Mais il est particulièrement éloquent qu'il cite aussi les graffitis et les tags de la culture visuelle Hip Hop et B-boy, racontée par Style 
Wars, (Fig.16) le film-documentaire réalisé par Tony Silver et Henry Chalfant en 1983 et présenté au Sundance Film Festival en 1984, année de la parution du livre de Chalfant et Martha Cooper, Subway Art, commenté par Danto en 1985 pour " The Nation ", et déjà objet de lectures importantes, comme celles de René Ricard et son marquant The Radiant Child du 1981, de Craig Castleman dans son livre Getting Up: Subway Graffiti in New York, paru en1982, de Hal Foster, et surtout de Jean Baudrillard qui dans son article séminal du 1976 Kool Killing, oppose les graffiti aux noms propres.

\section{Figure 15}

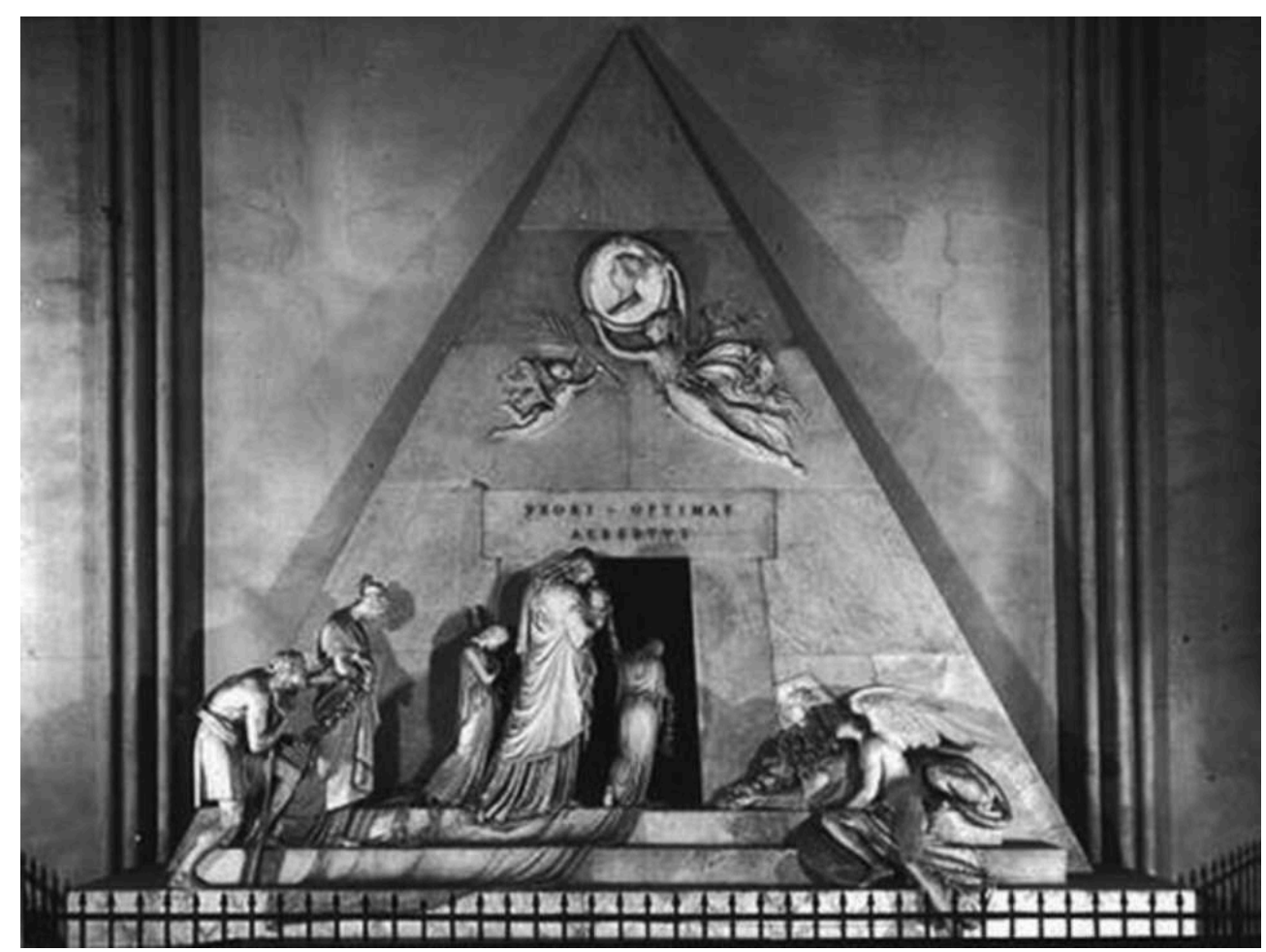

Antonio Canova, Monument funèbre à Marie Christine d'Autriche, 1798-1805, Augustinerkirche, Vienne. 
Figure 16

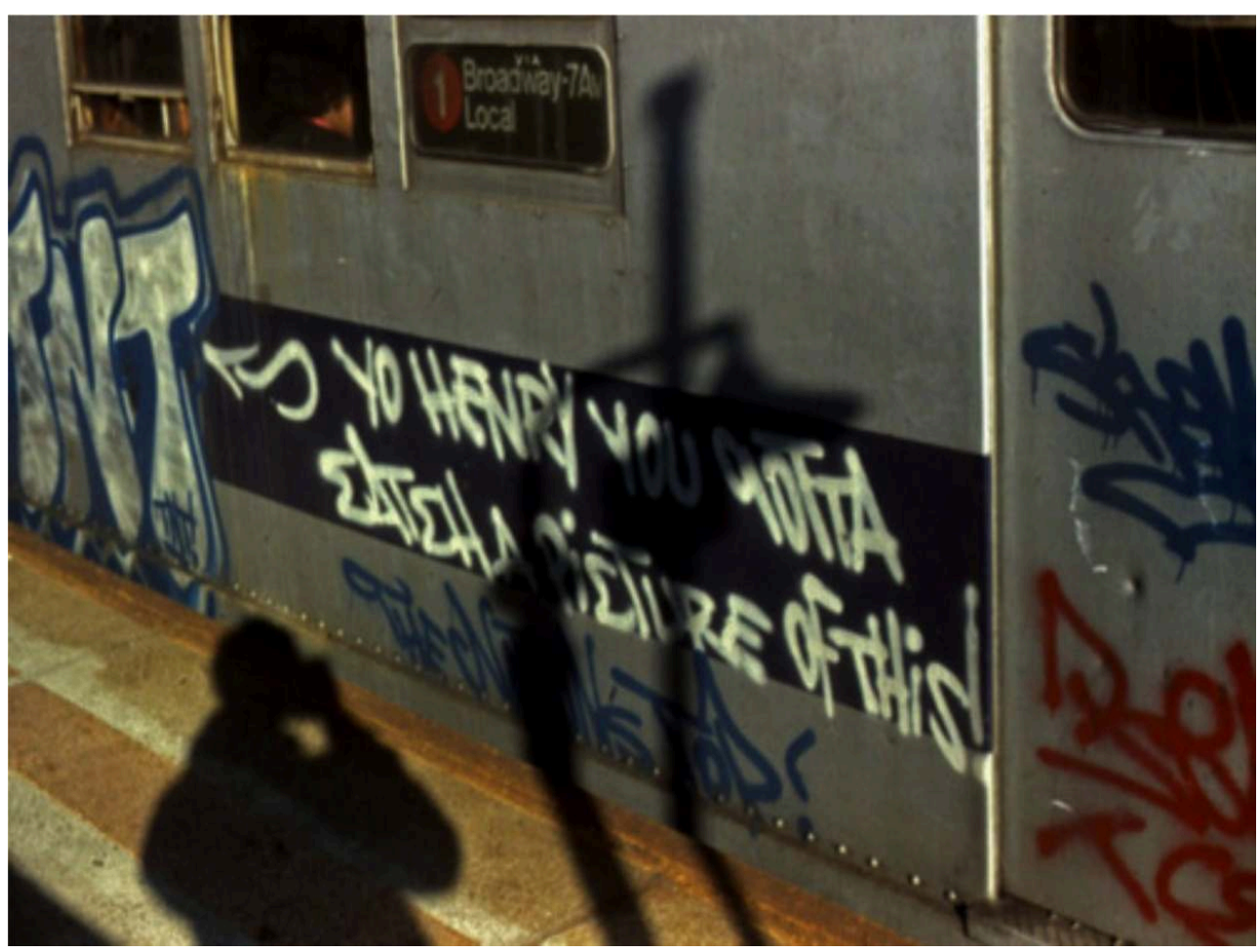

Tony Silver et Henry Chalfant, Style Wars, 1983, DVD (PBS).

L'enjeu méthodologique de cette batterie d'évocations visuelles est assez simple: la familiarité superficielle présupposée est, selon Danto, implicite chez les visiteurs du Mémorial, elle est bien synchronique et entièrement cadrée par l'habitude perceptuelle des habitants des métropoles nord-américaines, notamment acclimatés aux signes graphiques de la Street Art. Et pourtant, c'est justement cet air de famille aspectuel, apparemment troublant mais parfaitement cohérent avec la perspective sobrement pragmatiste et historiciste de Danto, qui aurait pu lui suggérer d'autres signes. En négatif, on penserait d'abord aux empreintes et aux taches qui parasitent les surfaces rouillées des anti-monuments de Serra, comme a montré Marshal Bermann dans un livre classique (All is Solid Melts Into Air: The Experience of Modernity, 1982), les innombrables dirties signs of time qui souillaient par exemple TWU à Canal street, ont marqué l'histoire même de New York. Selon le parti pris critique de Danto, ces traces attestent à la fois une de-artification de l'art public et une détérioration du lieu social et du contexte physique d'usage. Par ailleurs, comme a souligné récemment Nick Riggle dans un article qui pastiche Danto (Street Art: TheTransfiguration of the Commonplace), ces marques d'un usage routinier ne suffissent pas à soustraire ces artefacts monumentaux à l'Artworld et les insérer dans le Street Art ou dans le monde de la vie urbaine. Mais Danto aurait pu surtout citer les écritures imprimées sur la surface d'un autre monument funéraire, réalisé à peu près au moment où il élaborait son interprétation de l'art public discutant Serra et Maya Lin. 


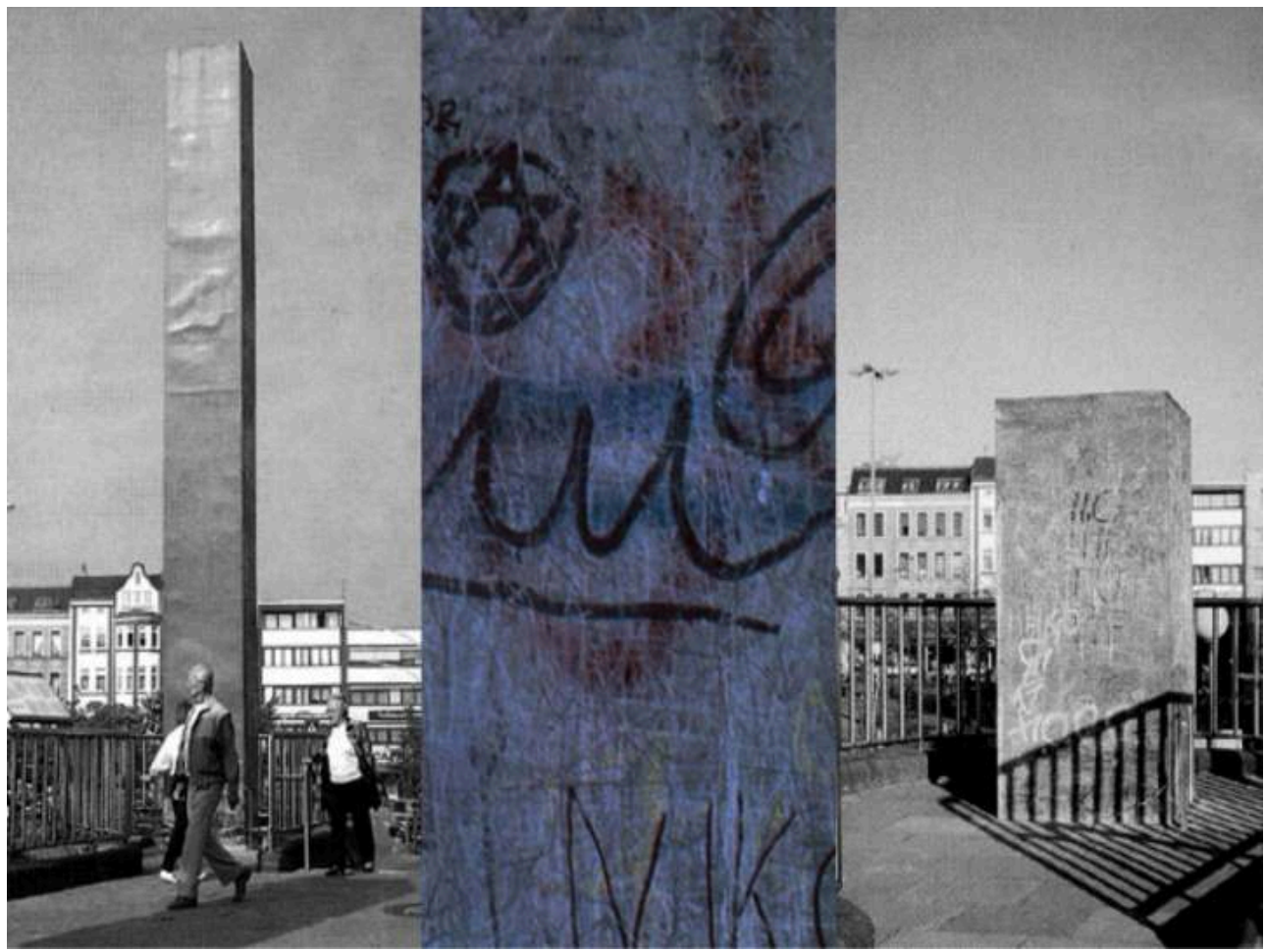

Jochen Gerz et Esther Shalev-Gerz, Monument contre le fascisme, 1986, Hambourg.

Ai-je dit "réalisé »? Le mot est peut-être trompeur. (Fig.17) Car le Monument contre le fascisme $^{18}$ de Jochen Gerz et Esther Shalev-Gerz, mis en place en 1986 dans un coin anonyme et ordinaire d'Hambourg, pas loin de la ville d'origine de Warburg, nie radicalement l'idée même du monument en tant que support mnémonique art-factuel durable et accessible à tout le monde. Le Monument contre le fascisme était un long bâton en acier zingué de 12 mètres, posé sur une base ; en plomb doux et gras pour pouvoir absorber toute peinture et dissuader les tagueurs, tout en permettant aux visiteurs et citoyens de prendre la parole seulement par un acte qui entaille, excave et creuse, bref par une action scripturaire. (Fig.18) Les visiteurs ont donc écrit, ils ont déposé leurs signatures et exprimé leurs idées, sensations, suggestions, commentaires, critiques, parfois superposant ces signes, lignes ou dessins à d'autres signes, guère aux noms déjà gravés, finalement méconnaissables ou illisibles. (Fig.19) Sauf que la tour s'enfonçait très lentement dans son socle, jusqu'à disparaître complètement, laissant vide le lieu de l'emplacement. Cet enterrement, déclenché par la pression exercée par les actions sur les surfaces subjectiles (on a calculé 70000 inscriptions), a duré cinq ans, ininterrompu et presque imperceptible jusqu'à un moment indéterminé, en novembre 1993. 
Figure 18

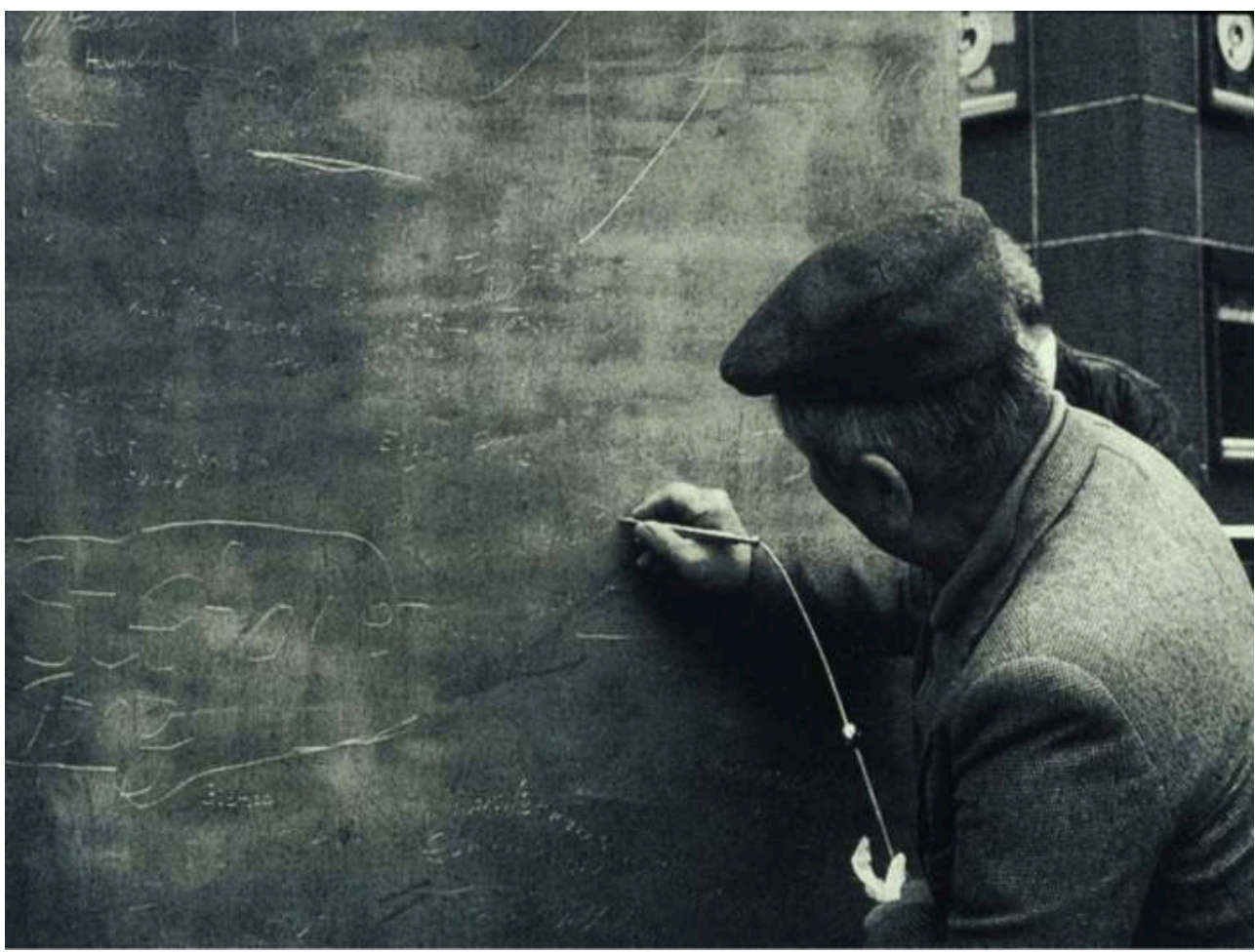

Jochen Gerz et Esther Shalev-Gerz, Monument contre le fascisme, 1986, Hambourg.

Figure 19

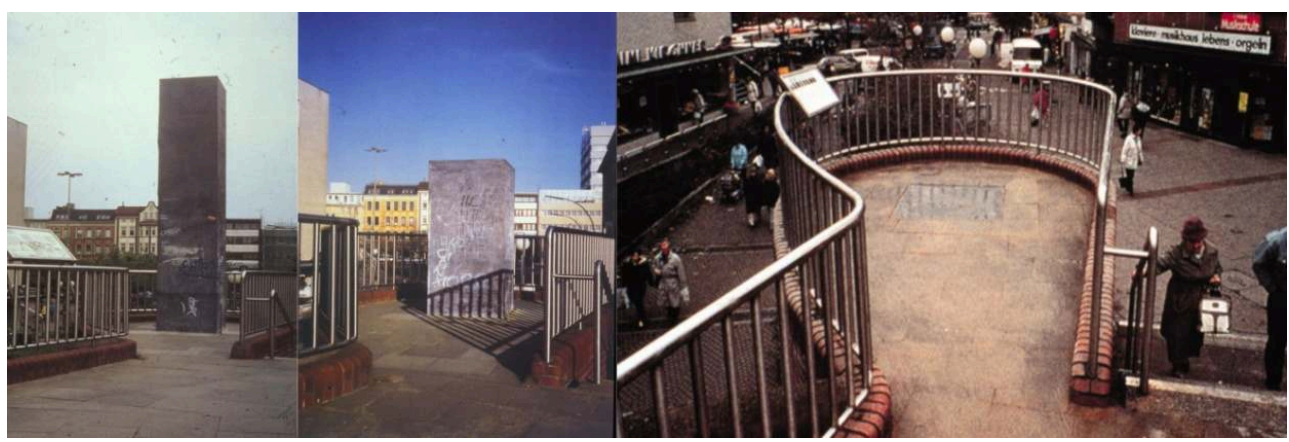

Maya Lin. Vietnam Veterans Memorial, 1982, Washington.

Ainsi, la participation réactive contre le Fascisme, activée chez le public devenu coauteur, se transformait en une prise de position contre affirmée par l'œuvre elle-même : tantôt contre la typologie formelle et symbolique de la monumentalité elle-même - la stèle, l'obélisque, ou le pilier-, ici invertie, tantôt contre les formes subreptices d'amnésie et d'oubli irrévocablement mises en place par une habitude perceptuelle et par une routine pragmatique. Prise de position qu'on dirait peut-être nietzschéenne car elle réalise la célèbre Umwertungaller Werte, l'inversion, et donc la transvaluation ou transmutation de toutes les valeurs - , ou dialectique au sens de la dialectique négative d'Adorno et Horkheimer, car adressée à affirmer par négation toute thèse ou position factuelle, toute positivité et Gestell, tout dispositif d'imposition et de cadrage. Ce n'est pas as par hasard qu'on a mis en parallèle cette prise de position contre-monumentale des Gerz avec la prise de parole parrhésiaste, avec le parler-franc des philosophes Grecs 
de l'Antiquité, qui énonce et dénonce sans médiations et compromis la vérité du pouvoir, qui lui fait face et se dresse contre lui.

Quoi qu'il en soit, la surface d'abord visible, verticale et frontale, de l'œuvre des Gerz devenait d'abord un texte, ou presque, car elle n'était qu'un simple support d'écritures transitoirement lisibles, une table assez malléable pour les marquages laissés par les spectateurs-usagers; on l'aurait dit une table de cire où, selon un modèle déjà existant chez Platon et Aristote, on peut consigner et déposer, fixer et garder réellement les signes d'une expérience esthétique et éthique d'un objet social singulier comme un monument. Sauf que cet artefact n'était pas, comme on aurait pu le croire, permanent et durable, mais, à l'opposé du Vietnam Veterans Memorial, il était en réalité programmé pour une « intemporalité limitée »- pour le dire avec Ulrich Krempel -, et destiné à une illisibilité et invisibilité sans appel.

Les phrases, les noms, les traces ou les graffitis, bref les écritures et les inscriptions sur ce médium destiné à être une archive encryptée et inaccessible, sont ainsi spéculaires aux noms propres gravés dans le Mémorial pour les Vétérans du Vietnam. Selon Maya Lin et la plupart des interprètes et des visiteurs, les deux surfaces en marbre noir poli évoqueraient les pages du Livre des Morts, sur lesquelles sont déposés, pour l'éternité, les noms des disparus. Danto ${ }^{19}$ résume à sa manière cette opinion : " les êtres [qui se reflètent sur le Mur] ne sont qu'apparences; seuls les noms des morts sont réels ». Autrement dit: seuls les noms, absolument identiques à eux-mêmes dans la transparence de l'inscription funéraire, sont ce que le Mémorial désigne et ce de quoi il est : son texte est un cénotaphe, une liste de noms propres. Un livre de noms, donc, et non pas de figures, certainement pas un livre d'images flottantes et aléatoires comme les reflets des autres monuments qui l'entourent et ceux des visiteurs eux-mêmes. Comme les êtres d'ombre du mythe platonicien, ces reflets ne sont rien d'autre que des apparences et des phantasmes. Ils incarnent les rêves ou les illusions politiques et militaires qui ont décrété d'abord la mort des vies dont les noms propres sont les marques singulières, célébrées et remémorées par la suite. (Fig.20) 
Figure 20

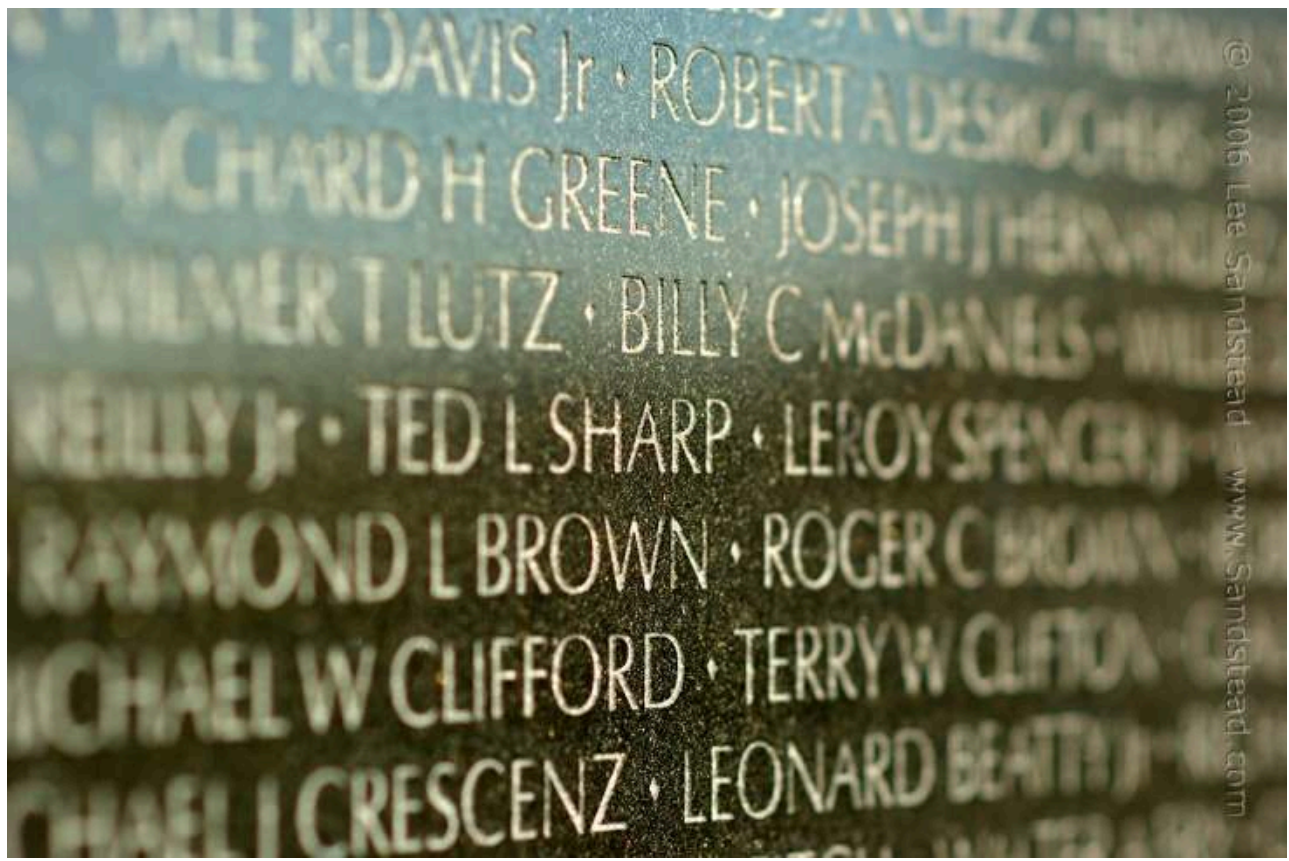

Maya Lin. Vietnam Veterans Memorial, 1982, Washington.

Figure 21

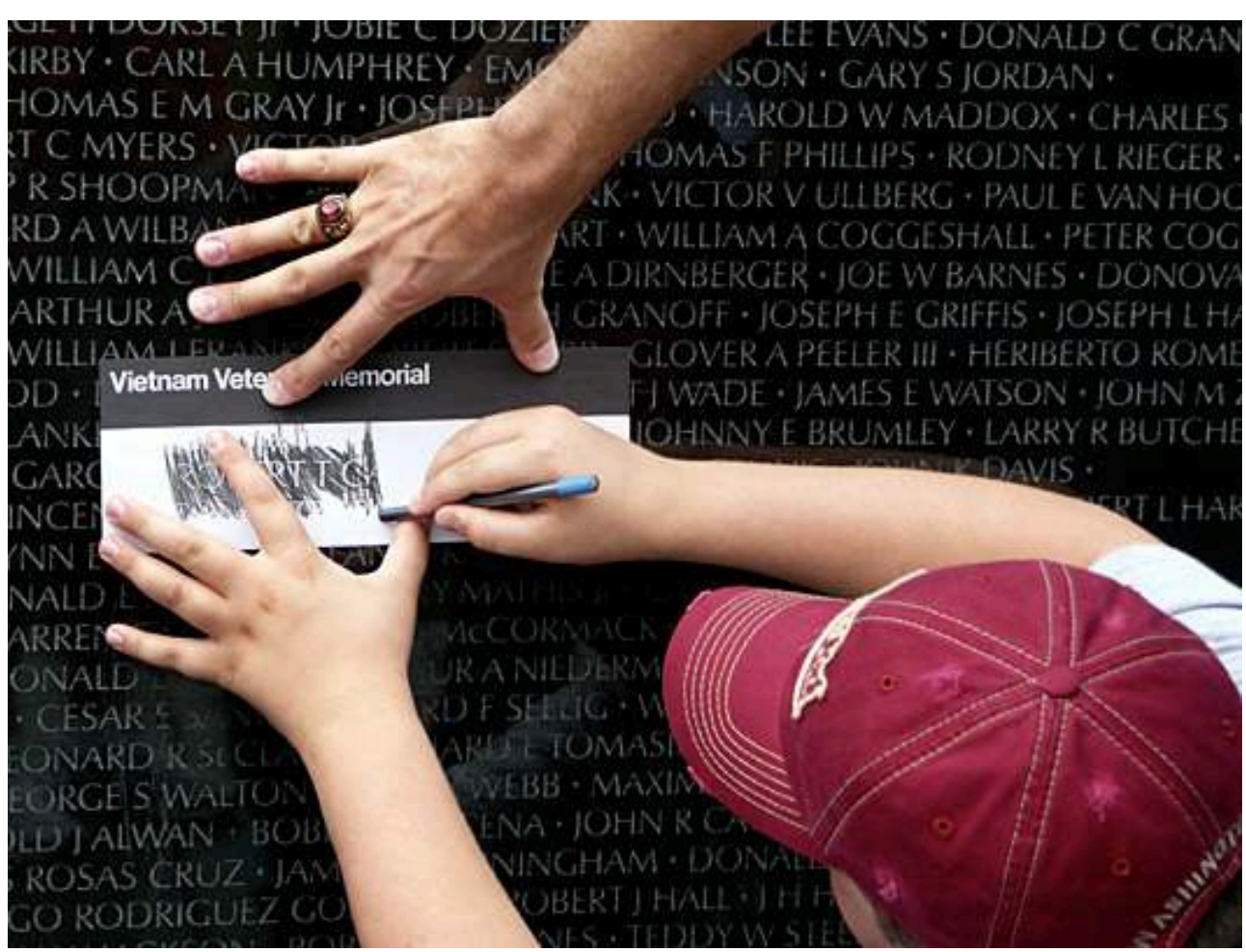

Maya Lin. Vietnam Veterans Memorial, 1982, Washington. 


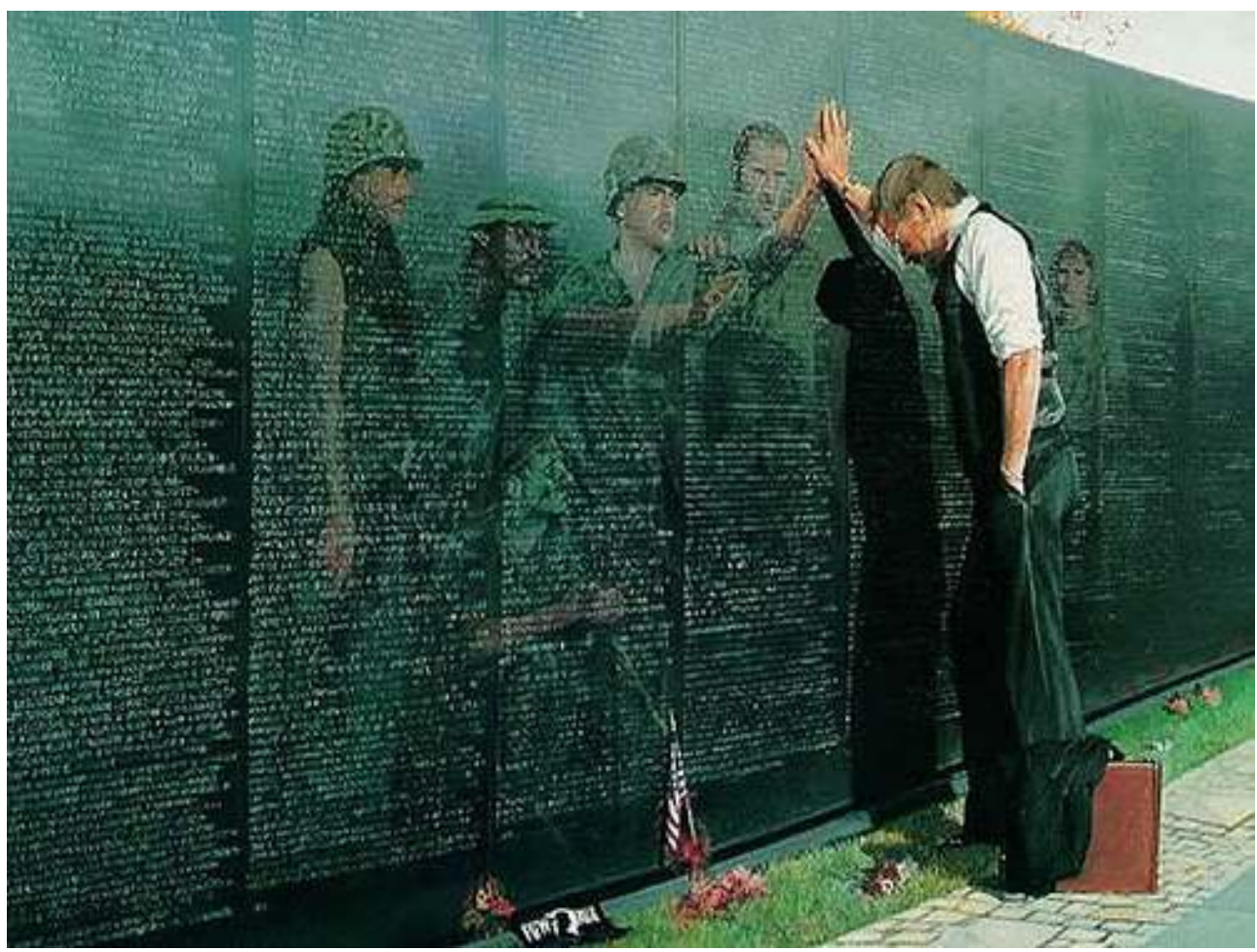

Maya Lin. Vietnam Veterans Memorial, 1982, Washington.

La lisibilité du texte du Mémorial est donc légitiment fondée sur la nature même du nom propre $^{20}$. La linguistique nous dit qu'un nom propre est absolument insubstituable et idéalement identique à soi-même ; (Fig.21) en fait, chaque nom propre est isolé dans la liste gravée du Mémorial, il est un signe-index copiable par frottage et transportable comme une carte de visite-ou, si j'ose le dire, comme un report card mémoriel durable - par les familles, les amis, les proches, les camarades ayant survécu. (Fig.22) $\mathrm{Au}$ contraire, la visibilité désincarnée des reflets, qui semblent apparaître aux visiteurs traumatisés comme les revenants des disparus et les faire revivre le chaos perceptif des batailles et des carnages au Vietnam, est, quant à elle, illusoire et fausse. Comme les eidola du mythe platonicien de la caverne (Res., 514b-520a) cité par Danto, il faut s'émanciper des reflets qui, comme des montages cinématographiques naturels, assemblent et mélangent les silhouettes du Washington Monument et du Lincoln Memorial, les masses mouvantes des arbres et des nuages, les profils des soldats et des infirmières en bronze, les monuments de Hart et de Goodacre, et les figures des visiteurs. Tous, comme suggère Danto ${ }^{21}$ évoquant les nymphéas de Monet, « semblent occuper le même espace ». 
Figure 23a

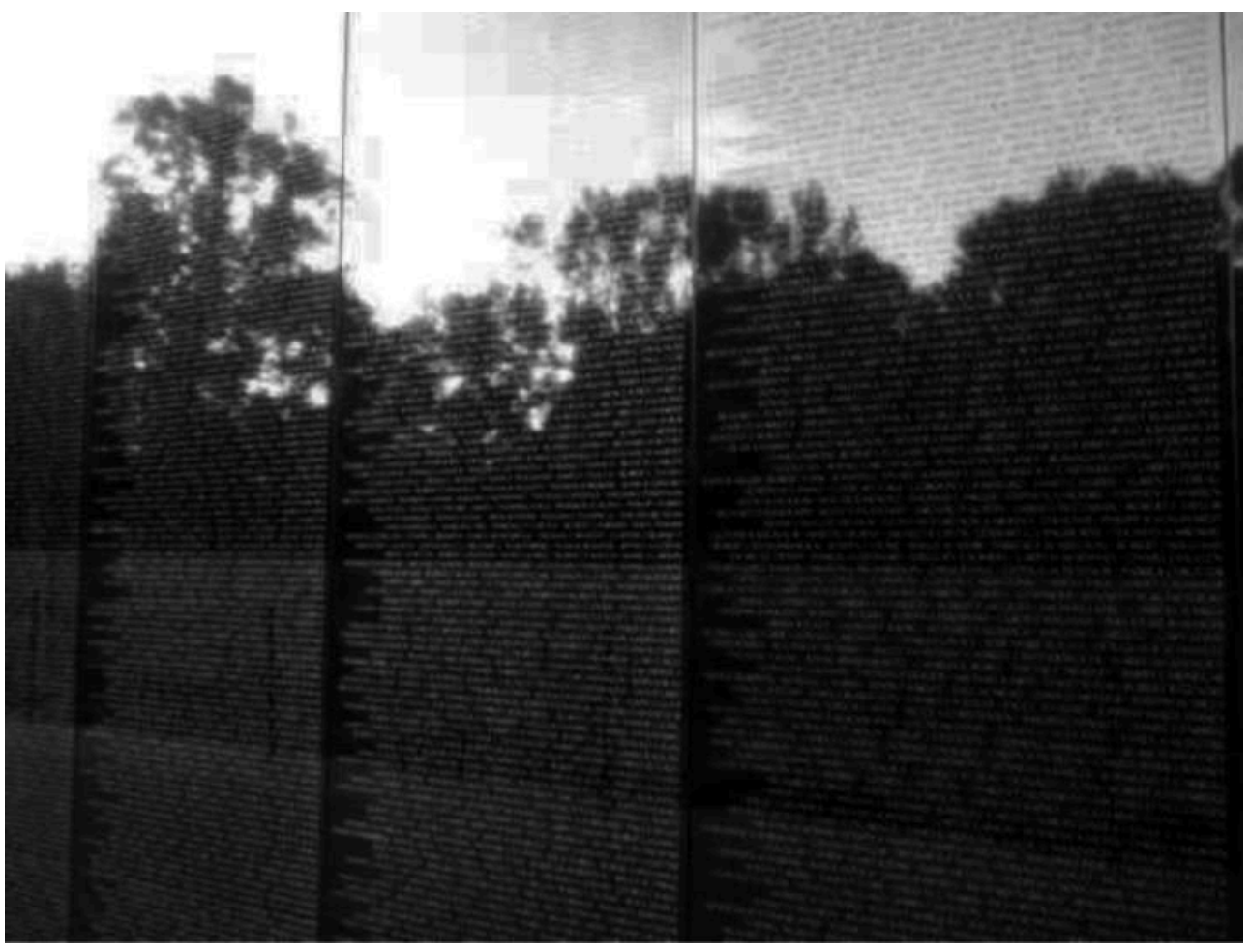

39 Maya Lin. Vietnam Veterans Memorial, 1982, Washington.

\section{Figure 23b}

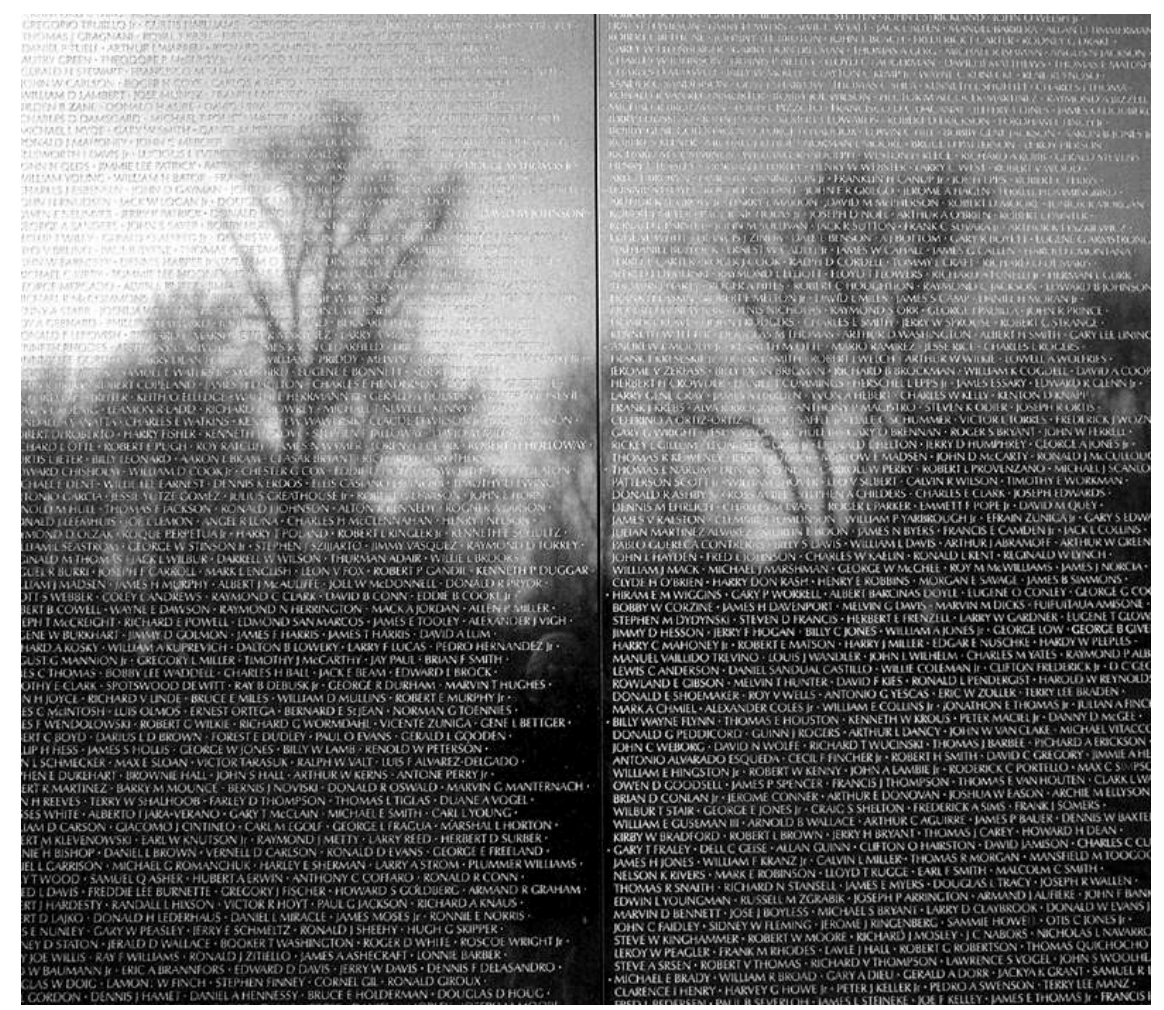

Maya Lin. Vietnam Veterans Memorial, 1982, Washington. 
Ces formes informes s'imposent en fait avec une grande puissance visuelle, synesthésique et presque hallucinatoire ${ }^{22}$ (Fig.23): la familiarité rassurante des architectures fondatrices de l'histoire s'évanouit comme dans un cauchemar éveillé, remplacée par la sauvagerie des aspects fantasmagoriques d'une nature ennemie et meurtrière ; les spectateurs sont frappés en pleine figure par les revenants ; le présent est habité par le passé, voire par l'archaïque de l'inconscient. "Comme s'il y avait une communauté des vivants et des morts ", dit justement Danto ${ }^{23}$. Ce qui revient à la surface du Mémorial - l'émergence singulière dont parle Foucault d'après Nietzsche -, est donc la contemporanéité, out of joints et presque extatique ou psychotique, du régime historique de la mémoire collective et du régime anachronique des traumas et des violences de l'histoire - en définitive de la mort. Finalement, le Meaning Embodiment, l'incorporation du sens intelligible et lisible, peut régresser jusqu'au Verkörperung, jusqu'à l'incorporation pathémique et sensible du visible, jusqu'à l'aveuglement par excès sensoriel et psychique.

41 Justement pour cela, les reflets sur le Mur, tantôt illusions désormais incroyables de la politique, tantôt apparitions fantasmatiques et survivantes des chocs de la guerre, exhibent finalement une ambiguïté de la mémoire ${ }^{24}$. Ambiguïté qui, pour Danto, devrait être dialectiquement - au sens de l'Aufhebung de Hegel-, supprimée et conservée, dépassée et accomplie par les noms propres à travers lesquels la communauté se reconnaît et se perpétue.

Or, l'anti-monument de Gerz s'oppose nettement à cette politique du nom propre dans Monument contre le fascisme, seuls l'oubli et l'anonymat des morts sans sépulture sont réels. Aucune identification, aucune auto-reconnaissance et transmission identitaire. Rien d'autre que du témoignage, à la fois à la première personne et le plus universel possible. (Fig.24) A la place du Monument disparu, enterré en tant que signe matériel et public qui aurait prétendu se substituer aux corps inconnus et innomés, une plaque dit en sept langues que c'est à chacun des citoyens et des visiteurs d'écrire ses nom et prénom à côté des informations sur les artistes: "Stelle sichgegen das Unrechterheben / Because nothing can permanently at our place rise up against injustice / Car rien ne peut durablement s'élever à notre place contre l'injustice ». Une signature collective, plus précisément une véritable prise de parole au pluriel, et dans plusieurs langues, engage ainsi tous les souscripteurs et s'adresse à tous les lecteurs à veiller et à prendre position, à se dresser à la place du monument enseveli ${ }^{25}$. L'œuvre est contre toute monumentalisation identitaire de la mémoire vivante et, justement pour cela, en tant que monument public; elle est d'elle-même soustraite à la vue. Texte illisible et image invisible, analogue à l'écriture originaire, noire, dont parle Barthes, le monument nie sa prédestination historique et son adresse éthico-esthétique, il rejette son usage social et politique préétabli. 
Figure 24

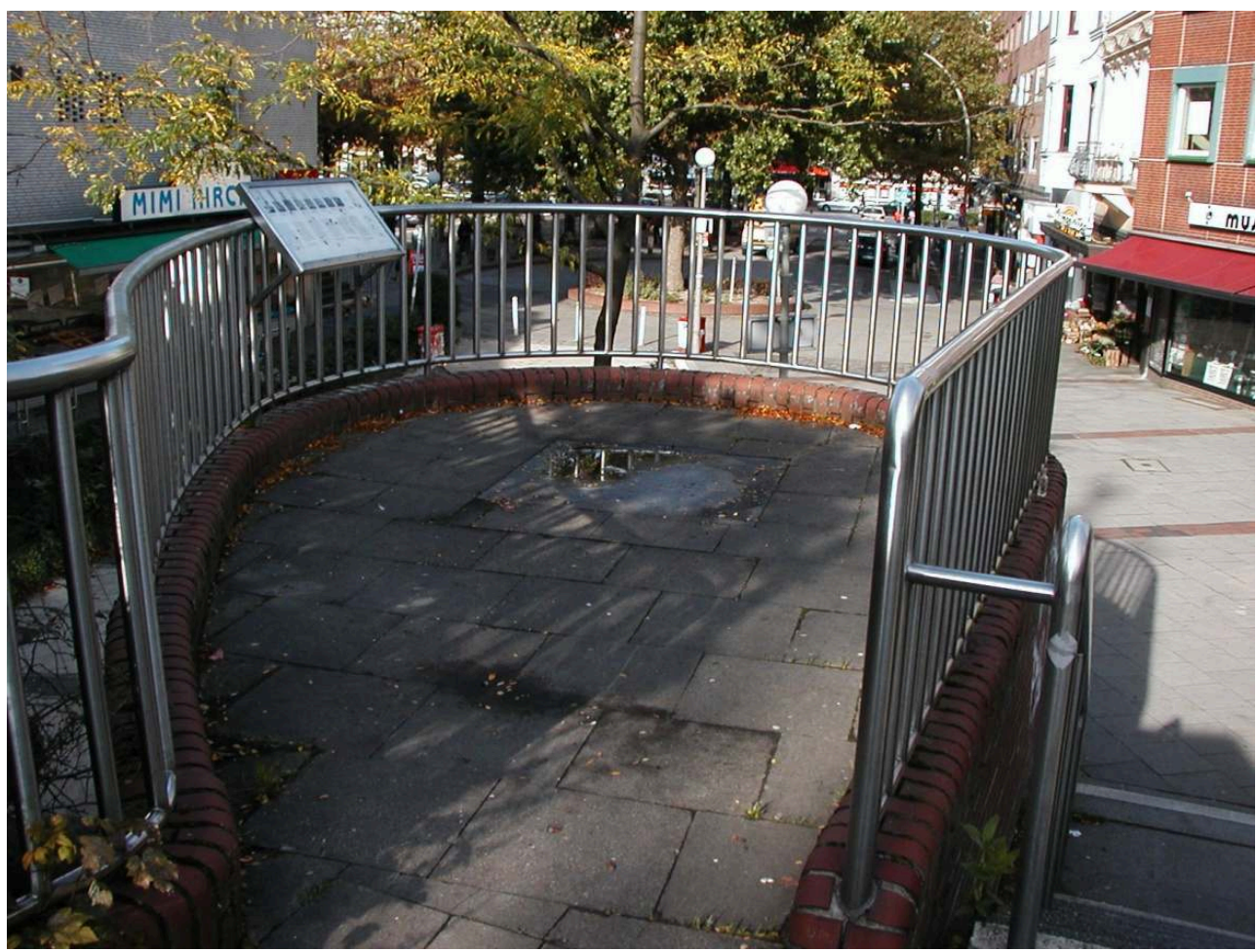

Jochen Gerz et Esther Shalev-Gerz, Monument contre le fascisme, 1986, Hambourg.

A mes yeux, le silence de Danto sur l'anti-écriture des Gerz et sur le dispositif de renversement de la nature, tantôt iconique tantôt textuelle, de l'art monumental est assez symptomatique. La Gegenschrift, ou l'anti-œuvre d'art public des artistes juifs, l'aurait peut-être obligé à problématiser la rhétorique politique de la beauté incarnée dans le Vietnam Veterans Memorial, "presque instantanément» ressentie, comme il l'affirme, et donc comprise.

Ce que Danto ne dit pas, c'est à nous d'essayer de le dénoncer, et justement à partir de son esthétique de l'art public. Car toute intelligibilité positive, y compris du Mémorial de Washington, est le résultat jamais définitif de la construction d'une identité collective, sociale, culturelle et politique, forcément opaque, c'est-à-dire continûment négociée et réalisée par des choix, des exclusions, des violences, des refoulements, des reniements, des oublis, et ainsi de suite. Tout cela, il faudrait l'interpréter comme partie intégrante du texte et du contexte des significations et des pratiques, des idéologies et des usages qui en décrètent et instituent la lisibilité et la visibilité ; ou bien inversement, l'absence ou la rature, l'expulsion ou l'omission dans les pages de l'historie. Cette opacité n'est pas prise en charge par la définition de la beauté morale avancée par Danto, qui ne donne pas assez de place à une véritable politique de la mémoire et de l'anti-mémoire, avec la beauté qui y est incorporée. 


\section{NOTES}

1. Merci à Jean-Patrice Courtois, Céline Flécheux, Marie Rebecchi et Tania Vladova pour les remarques et les relectures stimulantes de ces pages.

2. A. C. Danto, The Vietnam Veterans Memorial, (1985), in The Wake of Art: Criticism, Philosophy, and the Ends of Taste, selected with a critical introduction by G. Horowitz and T. Huhn, G + B Art International, Amsterdam, 1988, p. 154 et 156 la citation suivante : « given the textual nature of memorial art (of all art, when it comes to that) "; « Everything about [the Memorial] is part of a text $»$. Sauf autre indication, je traduis.

3. Pour une mise au point sur le débat et la bibliographie, je me permets de renvoyer au cinquième chapitre de Fantasmi dell'arte, Liguori, Naples, 2012, p. 105-130.

4. Yale University Press, Yale, 2013.

5. A. C. Danto, The Abuse of Beauty, Open Court Publishing, Peru (Ill.), 2003, p. $100:$ « Whatever the explanation on felt beauty of the Wall, it is understood with reference to the 'thought'. It is part of the meaning of the work. [...] In the Vietnam Veterans Memorial, the thought belongs to the work and explains the beauty. In natural beauty, the beauty is external to the thought, in the art the beauty is internal to the work $"$.

6. Ailleurs, Danto écrit: «La beauté d'une œuvre d'art est interne lorsqu'elle contribue à la signification de l'œuvre elle-même. [...] Si la beauté n'est pas interne à une œuvre d'art, elle est strictement sans signification, c'est-à-dire, avec les mots de Kant, qu'elle n'est que «beauté libre » et simple décoration. Bref, mon effort était rompre avec l'esthétique de la forme de Kant et Greenberg, et développer plutôt une esthétique de la signification. » Cf. A. C. Danto, Embodied Meanings as Aesthetical ideas, "Journal of Aesthetics and Art Criticism », vol. 65, no. 1, Winter 2007, p. 121-122: «The beauty of an artwork is internal when it contributes to the work's meaning. [...] who must acknowledge the meaning of this sacrifice by following their imperatives. If the beauty is not internal in a work of art it is, strictly speaking, meaningless, which means that it is, in Kant's terms, "free beauty" and mere decoration. In brief, my effort was to break away from the Kant-Greenberg aesthetic of form, and instead develop an aesthetics of meaning ".

7. A. C. Danto, Tilted arc and public art, (1985), in The State of the Art, Prentice Hall Press, New York, 1987, p. 90-94, et Richard Serra, (1986), ivi, p. 177-182. On remarquera que le premier texte, dans l'anthologie The Wake of Art, précède précisément l'article sur le Mémorial.

8. Je songe aux analyses classiques d'Aloïs Riegl et Cesare Brandi. Sur l'ensemble de ces problèmes, je me permets de renvoyer à L'art désœuvré, modes d'emploi : entre esthétique et théorie de la restauration, in J. Delaplace, P-H. Frangne, G. Mouëllic (sous la direction de), La pensée esthétique de Gérard Genette, PUR, Rennes, 2010, p. 133-154.

9. Cf. D. Novitz, «Rules, Creativity and Pictures: Wittgenstein's Lectures on Aesthetics ", in P. B. Lewis (ed.), Wittgenstein, Aesthetics and Philosophy, Ashgate, Aldershot, 2004, p. 55-72.

10. A.C. Danto, The Transfiguration of the Commonplace, Harvard University Press, Cambridge (Mass.), 1981, p. 169.

11. A. C. Danto, The Vietnam Veterans Memorial, loc. cit. : «We erect monuments so that we shall always remember, and build memorials so that we shall never forget. [...] Monuments commemorate the memorable and embody the myths of beginnings. Memorials ritualize remembrance and mark the reality of ends. [...] Very few nations erect monuments to their defeats, but many set up memorials to the defeated dead. Monuments make heroes and triumphs, victories and conquests, perpetually present and part of life. The memorial is a special precinct, extruded from life, a segregated enclave where we honor the dead. With monuments we honor ourselves ». On remarquera l'important changement de la première formulation de l'authorisation accordée de la Congress Joint Resolution à la Vietnam Veterans Memorial Fund : 
l'expression «erect a monument», devient «establish a memorial». Cf. R. Wagner-Pacifici, B. Schwartz, The Vietnam Veterans Memorial: Commemorating a Difficult Past, "The American Journal of Sociology ", vol. 97, no. 2, September 1991, p. 398.

12. R. Serra, "Richard Serra's Urban Sculpture. Interview by Douglas Crimp", (1980), in Writings/ Interviews, University of Chicago Press, Chicago, 1994, p. 134-135; cf. C. Nelson Blake, "An Atmosphere of Effrontery: Richard Serra, Tilted Arc and the Crisis of Public Art", in R. Wightman Fox, T.J. Jackson Lears (eds.), The Power of Culture : Critical Essays in American History, Chicago University Press, Chicago, 1993, p. 254 ss.

13. A. C. Danto, Tilted arc and public art, cit., p. 150 : « Public art is the public transfigured : it is us, in the medium of artistic transformation ".

14. A. C. Danto, The Abuse of the Beauty, cit., p. 100 : "The beauty of the Memorial is almost instantly felt, and then perhaps analyzed and explained by reference to the way the visitors, many of whom come to see the name of someone they knew or loved, and to do a rubbing of it to carry home, see themselves reflected in the same wall that carries the name of the dead, as if there were a community of the living and the dead, though death itself is forever ".

15. M. Foucault, Nietzsche, la généalogie, l'histoire, (1971), in Dits et Ecrits, D. Defert, F. Ewald et al (sous la direction de), Gallimard, Paris, 1994, p. 136-156, ainsi que pour les citations suivantes.

16. J'ose citer et interpoler F. Nietzsche, La Volonté de puissance, II, $§ 457$, trad. fr. par G. Bianquis. En 1965, Danto publie justement Nietzsche as Philosopher, Macmillan, New York.

17. Je songe bien sûr au fragment $[\mathrm{N} 2 \mathrm{a}, 3]$ du Livre des passages, trad. fr. par J. Lacoste, Éditions du Cerf, Paris, 1989, p. 480.

18. Pour une mise au point récente, J. Seipelt, Das 'Mahnmal gegen Faschismus, Krieg, Gewalt für Frieden und Menschenrechte' von Esther Shalev-Gerz und Jochen Gerz, GRIN Verlag, München, 2010.

19. A. C. Danto, The Vietnam Veterans Memorial, cit, p. 154 : «Still, the living are in it only as appearances. Only the names of the dead, on the surface, are real». Sur l'importance de la nomination pour les anti-monuments de la fin du XXème siècle, A. Zevi, Monumenti per difetto, Donzelli, Rome, 2014, p. 89-99.

20. Cf. K. Feldman, The shape of Mourning: Reading, Aesthetic cognition, and the Vietnam Veterans Memorial, «Word \& Image », vol. 19, no. 4, October-December 2003, pp. 296-304.

21. A. C. Danto, The Abuse of the Beauty, cit., p. 100. Sur cette lecture anti-greenberghienne de Monet, je dois ici survoler.

22. Cf. F. Cole, N. Watkins, S. Weidmann, The War Memorials Healing Environment : The Psychological Effect of the Vietnam Veterans Memorial on Vietnam War Combat Veterans' Post-traumatic Stress Disorder Symptoms, «Environment and Behavior », vol. 42, no. 3, May 2010, p. 351-75 ; N. Watkins Nicholas, Mirror, Mirror on the Wall: Ambiguous Place Attachment and the Vietnam Veterans Memorial, ThirtyEighth Annual Conference of the Environmental Design Research Association (EDRA), 2007, p. 38-50.

23. A. C. Danto, The Abuse of the Beauty, cit., p. 100.

24. Cf. L. Levi, "Window or Mirror: The Vietnam Veterans Memorial and the Ambiguity of Remembrance", in P. Homans Peter (ed.), Symbolic Loss: The Ambiguity of Mourning and Memory at Century's End, University Press of Virginia, Charlottesville, 2000, p. 105-125.

25. Cf. A. Pühringer, Interview with Jochen Gerz, Das Mahnmal bist du selbst, « untitled. The State of the Art », no. 3, Printemps 2012, p. 114-125. 


\section{RÉSUMÉS}

Arthur Danto affirme que le Vietnam Veterans Memorial de Washington incarne la beauté même de sa signification. Selon lui, cette «beauté interne » à l'œuvre d'art public est à la fois ressentie et lue, car elle est construite comme si elle était un texte et selon la rhétorique de l'enthymème, du syllogisme fondé sur une connaissance tacite et sur un lieu commun largement partagé et fortement probable. Pourtant, la référence à la pulchritudo adherens de Kant et à la philosophie de l'architecture est assez problématique. Danto évoque comme contre-exemple l'autoréférentialité illisible tantôt du Modernisme grenbergien, tantôt du minimalisme environnemental et antimonumental de Richard Serra, mais l'agentivité de la beauté réelle et partagée du Mémorial est hantée par une logique spectrale de l'incorporation. Ce qui s'incarne dans cette œuvre peut en fait toujours excéder la rhétorique de la reconnaissance supportée par le syllogisme et troubler le cadre identitaire de la communauté donnée, notamment nord-américaine. Les ombres des visiteurs réfléchies et mélangées aux noms sur le mur du Mémorial peuvent devenir des signesindex illisibles et pathétique, ou être aperçues comme une empreinte figurale, éphémère et inquiétante ; finalement, ces images désincarnées peuvent réaliser une incorporation médiale ce qu'on appellera avec Warburg Verkörperung - de l'arrière-monde, du royaume des morts.

Arthur Danto asserts that Maya Lin's Vietnam Veterans Memorial in Washington really embodies the beauty of his meaning. For him, the Memorial's "internal beauty" is felt and read because she is built as a text by the rhetoric of enthymeme, as a syllogism based on some tacit knowledges and highly probables communplaces. However, the relationship to the Kant's pulchritudo adhaerens and philosophy of architecture is not an easy one : Danto rejects as unreadable the self-referent formalism of Greenberg's Modernism and the environmental non-monumentality of Richard Serra's Minimalism, but the agency of the Memorial's shared internal beauty is haunted by an unquestioned spectral logic. Actually, the image embodiment in this work can always exceed the rhetoric of reconnaissance supported by the syllogistic rhetoric and disturb the identity framework of the nord-american community. The beholders' reflecting shape on the Memorial can become, finally, an unreadable and pathetic index, or be perceived as a figural imprint, ephemeral and troubling; these disembodied pictures can achieve a medial incarnation - e.g. a Verkörperung - of the underworld.

\section{INDEX}

Mots-clés : Anti-monuments, lisibilité, incorporation

Keywords : Counter-monuments, Readability, Embodiment

\section{AUTEUR}

\section{FILIPPO FIMIANI}

Université de Salerno / Paris I - ACTEProfesseur d'Esthétique à l'Université de Salerne, il est membre de la Società Italiana di Estetica (SIE), d'Æsthetica. Art et Philosophie (AES), de l'Institut Arts, Créations, Théories, Esthétique (ACTE) de l'Université Paris 1 Panthéon-Sorbonne-CNRS, du Centre Inter-Critique des Arts et des Discours sur les Arts (CICADA) de l'Université de Pau et des Pays de l'Adour, et d'autres centres et équipes de recherches en Italie et France. Il est codirecteur de la revue en-ligne Aisthesis. Pratiche, linguaggi e saperi dell'estetico. Parmi ses 
ouvrages, on rappelera Fantasmi dell'arte. Sei storie con spettatore (Naples 2012), Forme informi. Studi di poetiche del visuale (Gêne 2005), Poetica Mundi. (Palerme 2001) et Poetiche e genealogie (Naples 2000). Pour sa bibliographie complète : http://dsc.unisa.it/fimiani/ 